\title{
Exploring the potential biomarkers for prognosis of glioblastoma via Weighted Gene Co-expression Network Analysis
}

\author{
Mengyuan Zhang ${ }^{1}$, Zhike Zhou $^{2}$, Zhouyang Liu ${ }^{1}$, Fangxi Liu $^{1}$, Chuansheng Zhao ${ }^{\text {Corresp. } 1}$ \\ 1 Department of Neurology and Stroke Center, The First Hospital of China Medical University, Shenyang, China \\ 2 Department of Geriatrics, The First Hospital of China Medical University, Shenyang, China \\ Corresponding Author: Chuansheng Zhao \\ Email address: cszhao@cmu.edu.cn
}

Background: Glioblastoma (GBM) is the most common malignant tumor in the central system with a poor prognosis. Due to the complexity of its molecular mechanism, the recurrence rate and mortality rate of GBM patients are still high. Therefore, there is an urgent need to screen GBM biomarkers to prove the therapeutic effect and improve the prognosis. Results: We extracted data from GBM patients from the Gene Expression Integration Database (GEO), analyzed differentially expressed genes in GEO and identified key modules by weighted gene co-expression network analysis (WGCNA). GSE145128 data was obtained from the GEO database, and the darkturquoise module was determined to be the most relevant to the GBM prognosis by WGCNA $(r=-0.62, p=0.01)$. We performed enrichment analysis of gene ontology (GO) and Kyoto Encyclopedia of Genes and Genomes (KEGG) to reveal the interaction activity in the selected modules. Then Kaplan-Meier survival curve analysis was used to extract genes closely related to GBM prognosis. We used Kaplan-Meier survival curves to analyze the 139 genes in the darkturquoise module, identified four genes (DARS / GDI2 / P4HA2 / TRUB1) associated with prognostic GBM. Low expression of DARS/GDI2/TRUB1 and high expression of P4HA2 had a poor prognosis. Finally, we used tumor genome map (TCGA) data, verified the characteristics of hub genes through Co-expression analysis, Drug sensitivity analysis, TIMER database analysis and GSVA analysis. We downloaded the data of GBM from the TCGA database, the results of co-expression analysis showed that DARS/GDI2/P4HA2/TRUB1 could regulate the development of GBM by affecting genes such as CDC73/CDC123/B4GALT1/CUL2. Drug sensitivity analysis showed that genes are involved in many classic Cancer-related pathways including TSC/mTOR, RAS/MAPK.TIMER database analysis showed DARS expression is positively correlated with tumor purity(cor=0.125, $p=1.07 e-02)$ ), P4HA2 expression is negatively correlated with tumor purity (cor $=-0.279, p=6.06 \mathrm{e}-09$ ). Finally, GSVA analysis found that DARS/GDI2/P4HA2/TRUB1 gene sets are closely related to the 
occurrence of cancer. Conclusion: We used two public databases to identify four valuable biomarkers for GBM prognosis, namely DARS/GDI2/P4HA2/TRUB1, which have potential clinical application value and can be used as prognostic markers for GBM. 


\section{Exploring the potential biomarkers for prognosis of}

\section{2 glioblastoma via Weighted Gene Co-expression}

\section{Network Analysis}

4 Mengyuan Zhang ${ }^{1}$, Zhike Zhou ${ }^{2}$, Zhouyang Liu ${ }^{1}$, Fangxi Liu ${ }^{1}$ and Chuansheng Zhao ${ }^{1 *}$

51 Department of Neurology and Stroke Center, The First Hospital of China Medical

$6 \quad$ University, Shenyang 110000, PR China.

72 Department of Geriatrics, The First Affiliated Hospital, China Medical University,

8 Shenyang 110000 , PR China.

10 Corresponding Author: Chuansheng Zhao

11 The First Hospital of China Medical University, No. 155 Nanjing North Street, Heping District

12 Shenyang 110000, PR China

13 Email Adress: cszhao@cmu.edu.cn 


\section{Abstract:}

Glioblastoma (GBM) is the most common malignant tumor of the central system, with a poor prognosis.Due to the complexity of its molecular mechanism, the recurrence rate and mortality rate of GBM patients remain high. Therefore, screening of biomarkers for GBM is urgently needed to demonstrate therapeutic efficacy and improve prognosis. In this study, we extracted GBM patients' data from gene expression integration database (GEO), analyzed the differentially expressed genes in GEO by Weighted gene co-expression network analysis (WGCNA), constructed the co-expression network, and determined the correlation with the key recurrent modules of GBM. At the same time, based on Gene Ontology (GO) and Kyoto Encyclopedia of Genes and Genomes (KEGG), the selected modules were analyzed. Then four genes (DARS / GDI2 / P4HA2 / TRUB1) which are closely related to the prognosis of GBM were extracted by Kaplan-Meier survival curve analysis. The characteristics of these four genes were verified by tumor genome atlas (TCGA) data, Co-expression analysis, Drug sensitivity analysis, TIMER database analysis and Gene set variation analysis (GSVA) analysis. It was found that these four genes were differentially expressed genes in the initiation and progression of GBM, which could provide reference and basis for the observation of the clinical treatment and prognosis of GBM.

\section{Introduction}

Glioblastoma (GBM) is the most common primary neurogenic tumor, and the prognosis of most subtypes is poor(Tan et al. 2020). Despite of aggressive treatment strategies such as surgery followed by irradiation and chemotherapy, the prognoses of GBM patients remained unsatisfactory (Wu et al. 2020). According to the existing data, GBM patients have a survival of only 12-15 months after the standard treatment, with the 5-year survival rate of 3-5\% (Gong et al. 2020; Szopa et al. 2017). The main reasons for the poor prognosis of GBM are due to tumor metastasis and postoperative recurrence( $\mathrm{Tij}$ et al. 2021). Given that tumors invade the brain aggressively, GBM tumors can rarely be completely removed by surgery(Reichel et al. 2020). And the resulting network by GBM enables multicellular communication through microtubeassociated gap junctions, and increases tumor resistance to cell ablation and radiotherapy(Li et al. 2017a). Actively searching for biological markers related to the treatment and prognosis of GBM patients is of great significance for improving the survival rate of GBM patients.

In the past few decades, gene sequencing and bioinformatics analysis have been widely used for genetic variation screening at the gene level(Tingting et al. 2019), which helps us to identify differentially expressed genes (DEG) and functional pathways in the development of GBM. It has been found that the increased expression of SPRY2 mRNA indicates the decreased survival rate of GBM patients(Li et al. 2017a). Another study showed that the mRNA levels of NOTCH and Epidermal Growth Factor Receptor (EGFR) genes were increased in GBM tissues, which was related to the survival of patients(Irshad et al. 2015; Xing et al. 2015). However, most of 
52 these studies are single gene analysis, which may limit the analysis of the pathogenesis and

53 prognosis of GBM.

54 Weighted gene co-expression network analysis (WGCNA) is a platform to identify hub genes or therapeutic targets based on the interconnectivity of gene subsets and the association between gene subsets and phenotypes(Wang et al. 2020b; Zhang \& Horvath 2005). WGCNA can use the information of thousands of genes to identify the gene modules of interest and perform important association analysis on phenotypes. Recently, many journals have published relevant studies using WGCNA(Schafer et al. 2019; Wang et al. 2020b; Zhou et al. 2018).

In this study, we extracted four GBM related biomarkers (DARS / GDI2 / P4HA2 / TRUB1) by extracting data from GBM patients from the gene expression integrated database (GEO) and using WGCNA and Kaplan-Meier survival curves analysis. Then, we established GBM gene markers in the tumor genome atlas (TCGA), and confirmed the characteristics of these four genes by means of Co-expression analysis, Drug sensitivity analysis, TIMER database analysis , and GSVA analysis. In summary, our purpose is to find reliable biomarkers related to the prognosis of GBM by analyzing the relationship between DARS / GDI2 / P4HA2 / TRUB1 gene and GBM, so as to provide reference and basis for clinical treatment and prognosis observation of GBM.

\section{Materials and methods}

\section{Data information and construction of WGCNA}

The Series Matrix File data File of GSE145128 was downloaded from the NCBI GEO public database, which were contained 15 GBM patients and sets of transcriptional data, including untreated group $(n=7)$ and recurrent group $(n=8)$, for the construction of WGCNA co-expression network.

We constructed a weighted gene co-expression network to find co-expressed gene modules, and clarified the relationship between the gene network and phenotype and hub genes. The WGCNA$R$ package was used to construct a co-expression network of genes in the GSE145128 dataset, where the soft-thresholding power was set to 16 . The weighted adjacency matrix is converted into a topological overlap matrix (TOM) to estimate the network connectivity, and a hierarchical clustering method is used to construct a clustering tree structure of the TOM matrix. Different gene modules are represented by different cluster tree branches and colors. All genes are divided into multiple modules through gene expression patterns, and genes with similar expression patterns are divided into one module based on weighted correlation coefficients and expression patterns. 
86 In order to obtain the biological functions and signaling pathways involved in the interest module

87 of WGCNA, the Metascape database (www.metascape.org) was used for annotation and

88 visualization, and Gene Ontology (GO) analysis and Kyoto Encyclopedia of Genes and Genomes

89 (KEGG) pathway enrichment analyses were performed on the genes in the specific module. Min

90 overlap $\geq 3 \& \mathrm{p} \leq 0.01$ was considered statistically significant.

\section{$91 \quad$ Identifying Key Genes}

92 To determine key genes, the most important thing is whether they have an impact on tumor

93

94

95

96

97

98

99

100

101

102

103

104

105

106

107

108

109

110

111

112

113

114

115

116

117

118

prognosis. According to WGCNA theory, key genes have the highest connectivity in the module, which determines the biological significance of the module(Chen et al. 2019). So we think that key genes must exist in the interested module of WGCNA. Combined with the above two points, we analyzed the Kaplan-Meier survival rate of all genes in the interest module of WGCNA. We believe that the genes that can affect the prognosis of GBM patients are the key genes. And then,the next step is to explore and verify the specific molecular mechanism of key genes.

\section{Download and Pre-processing Data From TCGA}

TCGA database as the biggest cancer gene information database, including gene expression data, the miRNA expression data and copy number variation, DNA methylation, SNPS and other data. We downloaded the processed original mRNA expression data of GBM. A total of 159 specimens were collected(Supplementary table S1).

\section{Co-expression analysis}

The co-expression of the key genes were analyzed. The correlation coefficient filter condition was 0.3 and the p-value was 0.001 . After screening the genes with the most significant expression of key genes, the correlation analysis circles of key genes were plotted using "corrplot" and "circlize" packages.

\section{GSCALite and GDSC}

GSCALite is a set analysis platform for cancer genes. It integrates cancer genomics data of 33 cancer types from TCGA, drug response data from GDSC and CTRP, and normal tissue data from GTEx, and conducts gene set analysis in the data analysis process. Our study through this analysis was carried out on the key genes. Then base on the largest publicly available pharmacogenomics database (GDSC, the Genomics of Drug Sensitivity in Cancer, https://www.cancerrxgene.org/), we used the R packet "pRophetic" to predict the chemosensitivity of each tumor sample, and the estimated IC_(50) of each specific chemotherapeutic drug treatment was obtained by ridge regression. The prediction accuracy was measured by 10 -fold cross-validation with the GDSC training set. Select default values for all 
119 parameters, including "combats" to remove batch effects, "allSoldTumours" for tissue types, and

120 average values for summarizing repetitive gene expressions(Liu et al. 2018).

\section{TIMER database analysis}

122 TIMER is a website for systematically testing the molecular characteristics of tumor-immune

123 interactions(Li et al. 2017b). This website has incorporated 10,897 samples ranging from 32

124 different kinds of cancer types from the TCGA dataset(Shi et al. 2020). In this study, TIMER

125 was used to explore the relationship between key genes and the contents of immune cells and to

126 compare the infiltration levels between tumors with different somatic copy number changes of

127 key genes.

\section{Gene functional analysis}

129 GSVA uses a non-parametric and unsupervised method, and bypasses the traditional method of

130 explicitly modeling phenotypes in affluent scoring algorithms(Hanzelmann et al. 2013). By

131 comprehensively scoring the gene set of interest, GSVA converts the gene level change into the

132 pathway level change, and then judges the biological function of the sample. In this study, the

133 gene sets were downloaded from the Molecular signatures database (v7.0 version), and used the

134 algorithm of GSVA comprehensive score of each gene set, evaluating the potential biological

135 function change different samples.

\section{Statistical analysis}

137 All statistical analyses were performed in R language (version 3.6). All the statistical tests were

138 bilateral, and $\mathrm{p}<0.05$ was statistically significant.

\section{Result}

\section{Identification of gene co-expression modules}

142 The Series Matrix File data File of GSE145128 was downloaded from the NCBI GEO public

143 database. A total of 15 transcriptional data sets, including untreated group $(n=7)$ and recurrent

144 group $(\mathrm{n}=8)$, were used to construct the WGCNA co-expression network. In order to determine

145 whether the 15 samples in GSE145128 were suitable for network analysis, a sample dendrogram

146 and similar clinical features were studied. We confirmed that all samples were included in the

147 group (Fig. 1A). The soft-thresholding power was set as 16 for the subsequent construction of

148 co-expression network (Fig. 1B, Fig. 1C). The clustering tree structure of TOM matrix was

149 constructed by hierarchical clustering method. The different branches and colors represent

150 different gene modules (Fig. 1D). The network heatmap was used to analyze the interaction of 41 
151 modules (Fig. 1E). The results showed that each module was independent of each other,

152 indicating that each module was highly personalized and the gene expression of each module

153 was relatively independent.

\section{Correlation of modules and clinical traits}

155 In order to study the relationship between these modules and the prognosis of GBM, we

156 investigated the correlation between each module and the prognosis of GBM.We found that the

157 darkturquoise module had the highest correlation with disease relapse $(r=-0.62, p=0.01)$

158 (Fig. 2A, B ). We used Metascape to analyze the function and pathway of the darkturquoise

159 module. Metascape can identify the enrichment process in the gene list and the association

160 between enrichment processes $[14,15]$ by querying many databases,such as GO functional,

161 Hallmark Gene Sets, and KEGG pathways (Tripathi et al. 2015; Zheng et al. 2018). Based on

$162 \mathrm{GO}$ enrichment analysis, it was found that the co-expressed genes within the modules of interest

163 s mainly related to the steady state of cellular transition metal ion homeostasis, protein

164 hydroxylation, intrinsic apoptotic signaling pathway, ncRNA metabolic process(Fig. 2C). The

165 KEGG pathway analysis revealed that the co-expressed genes within the modules of interest was

166 mostly enriched in the 'Ferroptosis'(Fig. 2C). In addition, the enrichment processes were highly

167 connected and could be clustered into a complete network (Fig. 2C). These results indicated that

168 these functions were related in the occurrence and development of GBM.

\section{Identification of key genes in darkturquoise module}

170 According to the WGCNA theory, key genes have the highest connectivity in the module, which

171 determine the biological significance of the module and therefore influence the survial of

172 patients intensively(Chen et al. 2019). Therefore, we searched for hub genes in the darkturquoise

173 module.We analyzed the Kaplan-Meier survival curves of 139 genes in the darkturquoise

174 module. 14 genes with significant survival analysis results $(\mathrm{p}<0.05)$ were selected for

175 sequencing(Table. 1). Further determination of the key gene requires a combination of its

176 expression, typicality in previous studies, and previous research experience in our lab. Among

177 the 14 candidate genes we selected, most genes have been confirmed to be related to the

178 prognosis of GBM, such as: SPAG4, FKBP1B, DLEU1, PRKAR2B, NRL, CD24, GAS6; some

179 genes have not been clearly studied to be related to the occurrence of any known tumors, such as

180 CAMSAP2 ; the remaining genes have not been significantly expressed in GBM, such as

181 CORO6(Lo et al. 2009; Sun et al. 2021; Wang et al. 2019; Zhao et al. 2019). Finally, we found

182 that four genes (DARS/GDI2/P4HA2/TRUB1) had an impact on the survival rate of patients

183 with GBM, and were also confirmed to be related to tumorigenesis. At the same time, they were

184 not confirmed to participate in the occurrence and development of GBM, which met the

185 conditions for our further research (Fig. 3). Survival analysis showed that the patients with low

186 expression of DARS/GDI2/ TRUB1 and high expression of P4HA2 had poor prognosis. 
188

189

190

191

192

193

194

195

196

197

198

199

200

201

202

203

204

205

206

207

208

209

210

211

212

213

214

215

216

217

218

219

220

221

222

\section{Analysis of the co-expression of key genes}

It was clear that the key genes can affect the process of disease progression by regulating related genes. It can be assumed that DARS/GDI2/P4HA2/TRUB1 was associated with the most abundant pathways and genes and could regulate more biological processes. In order to assess the gene correlation of DARS / GDI2 / P4HA2 / TRUB1, we analyzed the co-expression of DARS/GDI2/P4HA2/TRUB1 through Pearson correlation analysis(cor $>0.3, \mathrm{p}<0.001$ ). We screened the 10 genes with the strongest correlation with the expression of DARS / GDI2 / P4HA2 / TRUB1, drew the correlation analysis map and heat map of DARS / GDI2 / P4HA2 / TRUB1 (Fig. 4A-4H), and found that the correlation between DARS and CDC73 was the highest, and the correlation between GDI2 and CDC123 was the highest. P4HA2 and B4GALT1 have the highest correlation, TRUB1 and CUL2 have the highest correlation (Fig. 4I-L). Then,we verified the modules of these four genes in WGCNA. We found that CDC73, CDC123 and CUL2 all exist in the darkturquoise module, which is consistent with DARS / GDI2 / P4HA2 / TRUB1(Supplementary table S2). Although B4GALT1 does not exist in the darkturquoise module, studies have confirmed that B4GALT1 can affect the development of GBM by regulating the apoptosis and autophagy(Wang et al. 2020a).Among them, CDC73 and CDC123 are cyclins of cell division(Sun et al.),B4GALT1 is one of seven $\beta$ - 1, 4-galactosyltransferases (B4GALT). CUL2 contributes to form E3 ubiquitin ligase that can recognize numerous substrates and is involved in a variety of cellular processes(Liu et al. 2019). These four genes have been shown to have a close relationship with many kinds of cancers(Cao et al. 2020a; Dou et al. 2020; Li et al. 2019a), such as thyroid carcinoma (Sarquis et al. 2019), breast cancer, etc.

\section{Cancer-related pathways and drug sensitivity analysis of key genes.}

First, we investigated the role of key genes in all well-known cancer-related pathways, as the following: TSC/mTOR, RTK, RAS/MAPK, PI3K/AKT, Hormone ER, Hormone AR, EMT, DNA Damage Response, Cell Cycle, Apoptosis pathways. The results found that DARS participated in the TSC/mTOR pathway activation; GDI2 was involved in Apoptosis, TSC/mTOR pathway activation; P4HA2 was involved in DNA Damage Response, EMT, Hormone AR, Hormone ER, RAS/MAPK and TSC/mTOR pathway; TRUB1 was involved in Apoptosis, DNA Damage Response, EMT, Hormone AR, PI3K/AKT and TSC/mTOR pathway (Fig. 5A). To investigate whether the expression of DARS / GDI2 / P4HA2 / TRUB1 in GBM had an impact on treatment (e.g. chemotherapies), we constructed a predictive model on six commonly used chemo drugs (i.e. AKT.inhibitor, Cisplatin, Dasatinib, Erlotinib, Gefitinib, and Gemcitabine) and confirmed that high expression of DARS was less sensitive to Cisplatin $(\mathrm{p}=0.00026)$ and Gemcitabine $(\mathrm{p}=0.0024)$, high expression of GDI2 was less sensitive to 
223 AKT.inhibitor $(\mathrm{p}=5.2 \mathrm{e}-05)$, Cisplatin( $\mathrm{p}=0.00067)$, Dasatinib $(\mathrm{p}=0.012)$ and

224 Gemcitabine $(\mathrm{p}=3.5 \mathrm{e}-06)$, low expression of P4HA2 was less sensitive to Cisplatin( $\mathrm{p}=0.0032)$,

225 and Gemcitabine $(\mathrm{p}=0.00017)$, and high expression of TRUB1 was less sensitive to

226 AKT.inhibitor( $\mathrm{p}=0.00043)$ (Fig.5B).

227

228

Immune cells infiltration analysis

229

In view of the obvious prognostic value of DARS/GDI2/P4HA2/TRUB1 gene, we used the

230 TIMER database to determine whether there was an association between tumor-infiltrating and

231 immune cells and DARS/GDI2/P4HA2/TRUB1 expression.Results showed that DARS

232 expression was positively correlated with tumor purity, P4HA2 and B cells (partial cor $=-0.239$,

233 $\mathrm{p}=7.89 \mathrm{e}-07$ ), P4HA2 and CD8+ T cells (partial cor $=-0.158, \mathrm{p}=1.19 \mathrm{e}-03$ ), TRUB1 and CD8+ T

234 cells (partial cor $=-0.206, p=1.87 \mathrm{e}-02$ ), DARS and neutrophils (partial cor $=0.245, \mathrm{p}=3.73 \mathrm{e}-07$ ), GDI2 and neutrophils (partial cor $=0.184, p=1.62 \mathrm{e}-04$ ), GDI2 and Dendritic cells (partial cor $=0.167, \mathrm{p}=6.21 \mathrm{e}-04$ ) P4HA2 and B cells (partial cor $=-0.239, \mathrm{p}=7.89 \mathrm{e}-07$ ), P4HA2 and CD8+

237 $\mathrm{T}$ cells (partial cor $=-0.158, \mathrm{p}=1.19 \mathrm{e}-03$ ), TRUB1 and $\mathrm{CD} 8+\mathrm{T}$ cells (partial cor $=-0.206, \mathrm{p}=1.87 \mathrm{e}-$ 02), DARS and neutrophils (partial cor $=0.245, \mathrm{p}=3.73 \mathrm{e}-07$ ), GDI2 and neutrophils (partial cor $=0.184, p=1.62 \mathrm{e}-04$ ), GDI2 and Dendritic cells (partial cor $=0.167, \mathrm{p}=6.21 \mathrm{e}-04$ ) (Fig. 6A). We also explored the correlation between tumor immune cell infiltration and somatic copy number alterations (SCNAs). The samples were divided into four types according to the copy number of genes. The distribution of infiltrating immune cells among the four types of samples was compared, as shown in Fig. 6B. We found that the various forms of mutations carried by the DARS / GDI2 / P4HA2 / TRUB1 gene can usually suppress immune infiltration, including $\mathrm{CD} 8+\mathrm{T}$ cells, neutrophils, dendritic cells, macrophages, CD4+T cells, and B cells. Also, we found that these four pivotal genes had a greater effect on immune infiltration than alterations in 247 the genes.

\section{Genomic alterations of DARS/GDI2/P4HA2/TRUB1 in GBM}

250

We then used the cBioPortal tool to determine the types and frequency of

251 DARS/GDI2/P4HA2/TRUB1 alterations based on DNA sequencing data from GBM patients.

252 The genetic variation rates of DARS/GDI2/P4HA2/TRUB1 ranged from $0 \%$ to $4 \%$ (DARS was $4 \%$, GDI2 was $1.4 \%$, P4HA2 was $4 \%$, TRUB1 was $0.0 \%$.) These alterations include Missense Mutation, mRNA High, mRNA Low, Amplification (AMP), and Deep Deletion.(Fig. 7) In view of this, DARS and P4HA2 show potentially stronger cancer-driving properties at a higher mutation frequency. In contrast, TRUB1 is genetically stable and could potentially act as a stable biomarker. 
259

260

261

262

263

264

265

266

267

268

269

270

271

272

273

274

275

276

277

278

279

280

281

282

283

284

285

286

287

288

289

290

291

292

293

294

\section{Gene functional analysis}

We downloaded the DARS/GDI2/P4HA2/TRUB1 gene sets from the Molecular signatures database (v7.0 version) and comprehensively evaluated the gene sets through GSVA. Our analysis showed that in the DARS gene set, 17 gene sets were up-regulated $(t>, 1)$ and 14 gene sets were down-regulated $(t<1)$. In GDI2, 13 gene sets were up-regulated and 21 gene sets were down-regulated. In P4HA2, 11 gene sets were up-regulated and 30 gene sets were downregulated. In TRUB1, 21 gene sets were down-regulated and 14 gene sets were down-regulated. (Fig. 8A - D).

\section{Discussion}

Due to the complex mechanisms of GBM, it is one of the most threatening CNS malignancies. Therefore, it is an urgent need to find biomarkers related to the occurrence and prognosis of GBM to reveal the possible pathogenesis or predict the prognosis of patients, and then develop personalized treatment plans for GBM patients. Based on gene sequencing technology, we have discovered some biological markers with predictive value for patients including GBM. However, the role of these markers are still limited. In order to better understand GBM, there is an urgent need to screen out more biomarkers to improve the efficacy of GBM treatment and prognosis.

GBM, as a highly heterogeneous tumor harboring multiple genetic alterations(Harter et al. 2014), molecular heterogeneity affects the effectiveness of single-molecule markers in predicting prognosis(Tonry 2020). At the same time, some studies have found that the high recurrence rate of GBM is related to the expression of strong proliferation genes of cells(Lara-Velazquez et al. 2020). And these processes usually involve multiple genes(Malik et al. 2020). Therefore, we believe that multi-gene markers have a higher predictive power for GBM prognosis than singlegene marker. We built a multi-gene markers model for predicting GBM prognosis, and validated the multi-gene markers model through strategies including training, testing, and independent cross-validation. The above strategies significantly improve the predictive ability of genetic markers (Li et al. 2019b).

In our research and analysis, the results of GO and KEGG analysis indicate that cell transition metal ion homeostasis, protein hydroxylation, intrinsic apoptotic signaling pathway and other processes may play an important role in GBM. Among them, transition metals are critical for many metabolic processes(Nelson \& N. 2014), and their steady state is vital to life. Aberrations in the cellular metal ion concentrations may lead to cell death and severe diseases such as cancer(Pi et al. 2020). Hydroxylation is a post-translational modification affecting protein stability, activity or interactome(Zurlo \& Zhang 2020). Many cancers are related to protein hydroxylation, such as breast cancer(Zurlo \& Zhang 2020), gastric cancer(Li et al. 2020), and prostate cancer(Della-Flora 
295

296

297

298

299

300

301

302

303

304

305

306

307

308

309

310

311

312

313

314

315

316

317

318

319

320

321

322

323

324

325

326

327

328

329

330

331

332

333

334

335

336

337

et al. 2020). For example, a study found that a set of enzymes PLOD1、PLOD2 and PLOD3 involved in the hydroxylation of lysine and stabilization of collagen by crosslinks, which upregulated expression in gastric cancer patients( $\mathrm{Li}$ et al. 2020). Similarly, intrinsic apoptotic signaling pathway can activate or inactivate multiple signaling pathways and inhibit multiple tumor suppressor genes, thereby promoting tumor progression. Almost all cancers involve intrinsic apoptotic signaling pathway, including renal cell carcinoma(Chae et al. 2020) and multiple myeloma(Chen et al. 2020a). Combined with the above results, we believe that DARS / GDI2 / P4HA2 / TRUB1 may be involved in these processes to affect the occurrence and development of GBM disease, which is also consistent with our Drug sensitivity analysis results. Among them, the DARS gene encodes the aspartyl-tRNA synthetase(Dominik et al. 2018), which pairs aspartate with its corresponding tRNA. Missense mutations in the gene encoding DARS can lead to leukocyte dystrophy, accompanied by a marked reduction in myelin sheath, abnormal movement and cognitive impairment (Fröhlich et al. 2018). There are no related reports about the relations between DARS and GBM.According to our research, DARS may participate in TSC/mTOR signaling, by regulating GBM cell growth process. GDI2 controls the activity of Rho GTPase's pathway to regulatory guanine nucleotide exchange factor and GTPase activating protein, and may play a role in tumor cell apoptosis. This is also in line with our results. At the same time, a recent study shows that RhoGDI2 suppresses lung metastasis in mice by reducing tumor versican expression and macrophage infiltration. The expression of P4HA2 increased in head and neck squamous cell carcinoma (HNSCC)(Kisoda et al. 2020), Oral Squamous Cell Carcinoma (OSCC)(Reis et al. 2020), cervical cancer(Cao et al. 2020b) and other cancers. Especially, we found that P4HA2 are markedly upregulated in cervical cancer tissues and upregulation of P4HA2 was associated with shorter overall survival (OS) and relapse-free survival (RFS)(Cao et al. 2020b). In GBM, we found that P4HA2 is mainly involved in the process of inhibiting DNA damage, and is also related to EMT, Hormone AR, Hormone ER, RAS / MAPK, TSC / mTOR and other pathways. TRUB1 mRNA is widely expressed in various human tissues (especially heart, skeletal muscle and liver), but there are few studies on its relationship with cancer(Zucchini et al. 2003). In our research, we analyzed that TRUB1 is mainly involved in Apoptosis, DNA damage, EMT, PI3K / AKT and other processes.

In the analysis of key genes co-expression, we found the four genes (CDC73 / CDC123 / B4GALT1 / CUL2) are most relevant to the expression of key genes and also related to the occurrence of many cancers. For example, CDC73 is a tumor suppressor, which can prevent cells from growing and dividing too fast or uncontrolled, and is closely related to parathyroid carcinoma(Cetani et al. 2019). CDC123 is a cell division cycle protein, and the regulatory effects of the entire cell cycle process can be stopped in one of the normal stages (G1, S, G2, M).CDC123 is highly expressed in choriocarcinoma(Hussain et al. 2018). B4GALT1 is one of the seven $\beta$-1,4-galactosyltransferase (beta4galt) genes. The $\beta 1,4$-galactosylation of glycans is very important for many biological events, including the development of cancer. In a variety of cancers, the B4GALTs family is associated with cancer cell proliferation, invasion, metastasis, and drug resistance.B4GALT1 is highly expressed in patients with lung adenocarcinoma(Zhang et al. 2019). CUL2is one of the seven members of Cullin family. It can participate in the regulation of cell cycle, proliferation, apoptosis, differentiation, gene expression, transcription regulation, signal transmission, damage repair, inflammation and immunity.CUL2 affects the

Peer) reviewing PDF | (2021:06:62268:4:0:NEW 17 Dec 2021) 
338 occurrence of renal cell carcinoma by promoting the substrate ubiquitination and

339 degradation(Liu et al. 2020).

340 Further TIMER analysis indicated that the immune system had a good effect on tumor

341

342

343

344

345

346

347

348

349

350

351

352

353

354

355

356

357

358

359

360

361

362

363

364

365

366

367

368

369

370

371

372

373

374

375

376 microenvironment, and that the mutations of DARS / GDI2 / P4HA2 / TRUB1 had important application value in tumor immunology. Finally, we conducted a comprehensive evaluation of gene sets using GSVA and we found that the DARS/GDI2/P4HA2/TRUB1 gene sets are closely related to the occurrence of cancer.For instance, the APICAL_JUNCTION in the DARS gene set is more common in highly differentiated epithelial cells, such as colon cancer cells(NairMenon et al. 2020).MITOTIC_SPINDLE in the GDI2 gene set, the mitotic spindle inhibitor is one of the most commonly used chemotherapeutics now(Bukowski et al. 2020). DNA_REPAIR in the P4HA2 gene set and ANGIOGENESIS in the TRUB1 gene set are also two important mechanisms of cancer development .

In recent years, with the GBM genes related to the occurrence and prognosis of feature recognition in many studies. Such as Chen X found the ASPMexpression pattern from the database showed that it is highly expressed in GBM tissue, and patients with high expression of ASPM have a poor prognosis(Chen et al. 2020b). Recently, a bioinformatic analysis of 123 GBM patients has established a 14-mRNA prognostic signature, which could be used to classify GBM patients into low and high risk groups(Arimappamagan et al. 2013).To our knowledge, the DARS/GDI2/P4HA2/TRUB1 that we identified are new GBM biomarkers because they have never been reported to be associated with the development and progression of $\mathrm{GBM}(\mathrm{Lu}$ et al. 2020). At the same time, compared with the traditional typing methods, the multi-gene markers model has many advantages, such as high prediction accuracy and personalized detection results(Albuquerque et al. 2012). Therefore, multi-gene markers have a good application prospect in clinical practice. In our study, we built and verified the characteristic of the four genes through analyzing the two independent data sets. More reasonable use of biometrics and multiple independent data sets of mutual verification makes our results more reliable.

However, our study had some limitations. Associated with disease, for example, age, race, sex, and some unknown prognostic factors may not be included in the model, which limits the prediction ability of the model. In the future, we plan to establish a more reasonable model of biological information analysis. Meanwhile, it should be acknowledged that the single gene analysis in this study does have limitations, and in future studies we will combine all the key genes or other factors together to find a biomarker with better sensitivity and accuracy using a multi-omics approach. In summary, our results had shown that DARS/GDI2/P4HA2/TRUB1 can be used as a new biological marker for GBM, which is related to the occurrence and prognosis of GBM, how to rationally apply various genetic characteristics at specific stages of GBM for diagnose and prediction of prognosis.

\section{Conclusion}

The molecular biological characteristics of GBM has changed the classification and treatment of tumors and become an important part of diagnosis and oncologic therapy. This study used

Peer) reviewing PDF | (2021:06:62268:4:0:NEW 17 Dec 2021) 
377 public databases to identify four valuable biomarkers for GBM prognosis, namely DARS / GDI2

378 / P4HA2 / TRUB1, which have potential and clinical application values to act as prognostic

379 markers of GBM.

380

\section{Acknowledgement}

382 None

383 Reference

384 Albuquerque AD, Kubisch I, Breier G, Stamminger G, Fersis N, Eichler A, Kaul S, and St?Lzel

385 U. 2012. Multimarker gene analysis of circulating tumor cells in pancreatic cancer patients: a

386 feasibility study. Oncology 82:3-10.

387 Arimappamagan A, Somasundaram K, Thennarasu K, Peddagangannagari S, Srinivasan H,

388 Shailaja BC, Samuel C, Patric IR, Shukla S, Thota B, Prasanna KV, Pandey P, Balasubramaniam

389 A, Santosh V, Chandramouli BA, Hegde AS, Kondaiah P, and Sathyanarayana Rao MR. 2013. A

390 fourteen gene GBM prognostic signature identifies association of immune response pathway and

391 mesenchymal subtype with high risk group. PLOS ONE 8:e62042. 10.1371/journal.pone.0062042

392 Bukowski K, Kciuk M, and Kontek R. 2020. Mechanisms of Multidrug Resistance in Cancer

393 Chemotherapy. Int J Mol Sci 21. 10.3390/ijms21093233

394 Cao Y, Han Q, Li J, Jia Y, and Shi H. 2020a. P4HA2 contributes to cervical cancer progression

395 via inducing epithelial-mesenchymal transition. Journal of Cancer 11:2788-2799.

396 Cao Y, Han Q, Li J, Jia Y, Zhang R, and Shi H. 2020b. P4HA2 contributes to cervical cancer

397 progression via inducing epithelial-mesenchymal transition. J Cancer 11:2788-2799.

398 10.7150/jca.38401

399 Cetani F, Marcocci C, Torregrossa L, and Pardi E. 2019. Atypical parathyroid adenomas:

400 challenging lesions in the differential diagnosis of endocrine tumors. Endocr Relat Cancer

401 26:R441-R464. 10.1530/ERC-19-0135

402 Chae IG, Song NY, Kim DH, Lee MY, Park JM, and Chun KS. 2020. Thymoquinone induces

403 apoptosis of human renal carcinoma Caki-1 cells by inhibiting JAK2/STAT3 through pro-

404 oxidant effect. Food Chem Toxicol 139:111253. 10.1016/j.fct.2020.111253

405 Chen G, Hu K, Sun H, Zhou J, Song D, Xu Z, Gao L, Lu Y, Cheng Y, Feng Q, Zhang H, Wang

406 Y, Hu L, Lu K, Wu X, Li B, Zhu W, and Shi J. 2020a. A novel phosphoramide compound,

407 DCZ0847, displays in vitro and in vivo anti-myeloma activity, alone or in combination with

408 bortezomib. Cancer Lett 478:45-55. 10.1016/j.canlet.2020.03.006

409 Chen L, Peng T, Luo Y, Zhou F, Wang G, Qian K, Xiao Y, and Wang X. 2019. ACAT1 and

410 Metabolism-Related Pathways Are Essential for the Progression of Clear Cell Renal Cell

411 Carcinoma (ccRCC), as Determined by Co-expression Network Analysis. Front Oncol 9:957.

$412 \quad 10.3389 /$ fonc. 2019.00957

413 Chen X, Huang L, Yang Y, Chen S, Sun J, Ma C, Xie J, Song Y, and Yang J. 2020b. ASPM 
414 promotes glioblastoma growth by regulating G1 restriction point progression and Wnt-beta-

415 catenin signaling. Aging (Albany NY) 12:224-241. 10.18632/aging.102612

416 Della-Flora A, Wilde ML, Pinto IDF, Lima EC, and Sirtori C. 2020. Degradation of the

417 anticancer drug flutamide by solar photo-Fenton treatment at near-neutral $\mathrm{pH}$ : Identification of

418 transformation products and in silico (Q)SAR risk assessment. Environ Res 183:109223.

419 10.1016/j.envres.2020.109223

420 Dominik F, Suchowerska AK, Carola V, He R, Ernst W, Georg VJ, Cas S, Thomas F, Housley

421 GD, and Matthias K. 2018. Expression Pattern of the Aspartyl-tRNA Synthetase DARS in the

422 Human Brain. Frontiers in Molecular Neuroscience 11:81-.

423 Dou B, Jiang Z, Chen X, Wang C, and Sheng G. 2020. Oncogenic Long Noncoding RNA

424 DARS-AS1 in Childhood Acute Myeloid Leukemia by Binding to microRNA-425. Technology

425 in Cancer Research \& Treatment 19:153303382096558.

426 Fröhlich D, Suchowerska AK, Voss C, He R, Wolvetang E, von Jonquieres G, Simons C, Fath T, 427 Housley GD, and Klugmann M. 2018. Expression Pattern of the Aspartyl-tRNA Synthetase 428 DARS in the Human Brain. Frontiers in Molecular Neuroscience 11. 10.3389/fnmol.2018.00081

429 Gong Z, Hong F, Wang H, Zhang X, and Chen J. 2020. An eight-mRNA signature outperforms 430 the lncRNA-based signature in predicting prognosis of patients with glioblastoma. BMC Med 431 Genet 21:56. 10.1186/s12881-020-0992-7

432 Hanzelmann S, Castelo R, and Guinney J. 2013. GSVA: gene set variation analysis for 433 microarray and RNA-seq data. BMC Bioinformatics 14:7. 10.1186/1471-2105-14-7

434 Harter D, Wilson T, and Karajannis M. 2014. Glioblastoma multiforme: State of the art and 435 future therapeutics. Surgical Neurology International 5. 10.4103/2152-7806.132138

436 Hussain S, Saxena S, Shrivastava S, Mohanty AK, Kumar S, Singh RJ, Kumar A, Wani SA, 437 Gandham RK, Kumar N, Sharma AK, Tiwari AK, and Singh RK. 2018. Gene expression 438 profiling of spontaneously occurring canine mammary tumours: Insight into gene networks and 439 pathways linked to cancer pathogenesis. PLoS ONE 13:e0208656.

440 10.1371/journal.pone.0208656

441 Irshad K, Mohapatra SK, Srivastava C, Garg H, Mishra S, Dikshit B, Sarkar C, Gupta D, 442 Chandra PS, Chattopadhyay P, Sinha S, and Chosdol K. 2015. A combined gene signature of 443 hypoxia and notch pathway in human glioblastoma and its prognostic relevance. PLoS ONE 444 10:e0118201. 10.1371/journal.pone.0118201

445 Kisoda S, Shao W, Fujiwara N, Mouri Y, Tsunematsu T, Jin S, Arakaki R, Ishimaru N, and 446 Kudo Y. 2020. Prognostic value of partial EMT-related genes in head and neck squamous cell 447 carcinoma by a bioinformatic analysis. Oral Dis. 10.1111/odi.13351

448 Lara-Velazquez M, Zarco N, Carrano A, Phillipps J, and Guerrero-Cazares H. 2020. 543: 449 Cerebrospinal Fluid-Responsive Factor SERPINA3 Promotes Proliferation, Migration and 450 Invasion of Glioblastoma. 543: Cerebrospinal Fluid-Responsive Factor SERPINA3 Promotes 451 Proliferation, Migration and Invasion of Glioblastoma.

452 Li C, Tan J, Chang J, Li W, Liu Z, Li N, and Ji Y. 2017a. Radioiodine-labeled anti-epidermal 
453 growth factor receptor binding bovine serum albumin-polycaprolactone for targeting imaging of glioblastoma. Oncol Rep 38:2919-2926. 10.3892/or.2017.5937

455

456

457

458

459

460

461

462

463

464

465

466

467

468

469

470

471

472

473

474

475

476

477

478

479

480

481

482

483

484

485

486

487

488

489

490

Li Q, Wang Q, Zhang Q, Zhang J, and Zhang J. 2019a. Collagen prolyl 4-hydroxylase 2 predicts worse prognosis and promotes glycolysis in cervical cancer. American Journal of Translational Research 11:6938-6951.

Li SS, Lian YF, Huang YL, Huang YH, and Xiao J. 2020. Overexpressing PLOD family genes predict poor prognosis in gastric cancer. J Cancer 11:121-131. 10.7150/jca.35763

Li T, Fan J, Wang B, Traugh N, Chen Q, Liu JS, Li B, and Liu XS. 2017b. TIMER: A Web Server for Comprehensive Analysis of Tumor-Infiltrating Immune Cells. Cancer Research 77:e108-e110. 10.1158/0008-5472.Can-17-0307

Li W, Lu J, Ma Z, Zhao J, and Liu J. 2019b. An Integrated Model Based on a Six-Gene Signature Predicts Overall Survival in Patients With Hepatocellular Carcinoma. Front Genet 10:1323. 10.3389/fgene.2019.01323

Liu A, Zhang S, Shen Y, Lei R, and Wang Y. 2019. Association of mRNA expression levels of Cullin family members with prognosis in breast cancer. Medicine 98 .

$10.1097 / \mathrm{md} .0000000000016625$

Liu CJ, Hu FF, Xia MX, Han L, Zhang Q, and Guo AY. 2018. GSCALite: a web server for gene set cancer analysis. Bioinformatics 34:3771-3772. 10.1093/bioinformatics/bty411

Liu X, Zurlo G, and Zhang Q. 2020. The Roles of Cullin-2 E3 Ubiquitin Ligase Complex in Cancer. Adv Exp Med Biol 1217:173-186. 10.1007/978-981-15-1025-0_11

Lo HW, Zhu H, Cao X, Aldrich A, and Ali-Osman F. 2009. A novel splice variant of GLI1 that promotes glioblastoma cell migration and invasion. Cancer Res 69:6790-6798. 10.1158/00085472.CAN-09-0886

Lu WC, Xie H, Yuan C, Li JJ, and Wu AH. 2020. Identification of potential biomarkers and candidate small molecule drugs in glioblastoma. Cancer Cell International 20.

Malik V, Garg S, Afzal S, Dhanjal JK, and Wadhwa R. 2020. Bioinformatics and Molecular Insights to Anti-Metastasis Activity of Triethylene Glycol Derivatives. International Journal of Molecular Sciences 21.

Nair-Menon J, Daulagala AC, Connor DM, Rutledge L, Penix T, Bridges MC, Wellslager B, Spyropoulos DD, Timmers CD, Broome AM, and Kourtidis A. 2020. Predominant Distribution of the RNAi Machinery at Apical Adherens Junctions in Colonic Epithelia Is Disrupted in Cancer. Int J Mol Sci 21. 10.3390/ijms21072559

Nelson, and N. 2014. Metal ion transporters and homeostasis. Embo Journal 18:4361-4371.

Pi H, Wendel BM, and Helmann JD. 2020. Dysregulation of Magnesium Transport Protects Bacillus subtilis against Manganese and Cobalt Intoxication. J Bacteriol 202. 10.1128/JB.0071119

Reichel D, Sagong B, Teh J, Zhang Y, and Perez JM. 2020. Near Infrared Fluorescent Nanoplatform for Targeted Intraoperative Resection and Chemotherapeutic Treatment of

Peer) reviewing PDF | (2021:06:62268:4:0:NEW 17 Dec 2021) 
491

492

493

494

495

496

497

498

499

500

501

502

503

504

505

506

507

508

509

510

511

512

513

514

515

516

517

518

519

520

521

522

523

524

525

526

527

528

529

Glioblastoma. ACS Nano XXXX.

Reis PP, Tokar T, Goswami RS, Xuan Y, Sukhai M, Seneda AL, Moz LES, Perez-Ordonez B, Simpson C, Goldstein D, Brown D, Gilbert R, Gullane P, Irish J, Jurisica I, and Kamel-Reid S. 2020. A 4-gene signature from histologically normal surgical margins predicts local recurrence in patients with oral carcinoma: clinical validation. Sci Rep 10:1713. 10.1038/s41598-020$58688-\mathrm{y}$

Sarquis M, Marx SJ, Beckers A, Bradwell AR, Simonds WF, Bicalho MAC, Daly AF, Betea D, Friedman E, and De Marco L. 2019. Long-term remission of disseminated parathyroid cancer following immunotherapy. Endocrine 67:204-208. 10.1007/s12020-019-02136-z

Schafer ST, Paquola ACM, Stern S, Gosselin D, Ku M, Pena M, Kuret TJM, Liyanage M, Mansour AA, Jaeger BN, Marchetto MC, Glass CK, Mertens J, and Gage FH. 2019. Pathological priming causes developmental gene network heterochronicity in autistic subject-derived neurons. Nature Neuroscience 22:243-255. 10.1038/s41593-018-0295-x

Shi S, Ye S, Mao J, Ru Y, Lu Y, Wu X, Xu M, Zhu T, Wang Y, Chen Y, Tang X, and Xi Y. 2020. CMA1 is potent prognostic marker and associates with immune infiltration in gastric cancer. Autoimmunity: 1-8. 10.1080/08916934.2020.1735371

Sun LW, Kao SH, Yang SF, Jhang SW, Lin YC, Chen CM, and Hsieh YH. 2021. Corosolic Acid Attenuates the Invasiveness of Glioblastoma Cells by Promoting CHIP-Mediated AXL Degradation and Inhibiting GAS6/AXL/JAK Axis. Cells 10. 10.3390/cells10112919

Sun W, Kuang XL, Liu YP, Tian LF, Yan XX, and Xu W. Crystal structure of the N-terminal domain of human CDC73 and its implications for the hyperparathyroidism-jaw tumor (HPT-JT) syndrome. Scientific Reports.

Szopa W, Burley TA, Kramer-Marek G, and Kaspera W. 2017. Diagnostic and Therapeutic Biomarkers in Glioblastoma: Current Status and Future Perspectives. Biomed Res Int 2017:8013575. 10.1155/2017/8013575

Tan AC, Ashley DM, López G, Malinzak M, and Khasraw M. 2020. Management of glioblastoma: State of the art and future directions. CA A Cancer Journal for Clinicians 70.

Tij A, Pr A, Acbc D, Is A, e FMMbcd, Rk F, Tka G, Dszbc H, Mkbc D, and Rm I. 2021. Frontiers in the treatment of glioblastoma: Past, present and emerging - ScienceDirect. Advanced Drug Delivery Reviews.

Tingting, Long, Zijing, Liu, Xing, Zhou, Shuang, Yu, Hui, and Tian. 2019. Identification of differentially expressed genes and enriched pathways in lung cancer using bioinformatics analysis. Molecular Medicine Reports.

Tonry C. 2020. Clinical proteomics for prostate cancer: understanding prostate cancer pathology and protein biomarkers for improved disease management. Clinical Proteomics 17.

Tripathi S, Pohl Marie O, Zhou Y, Rodriguez-Frandsen A, Wang G, Stein David A, Moulton Hong M, DeJesus P, Che J, Mulder Lubbertus CF, Yángüez E, Andenmatten D, Pache L, Manicassamy B, Albrecht Randy A, Gonzalez Maria G, Nguyen Q, Brass A, Elledge S, White M, Shapira S, Hacohen N, Karlas A, Meyer Thomas F, Shales M, Gatorano A, Johnson 
530 Jeffrey R, Jang G, Johnson T, Verschueren E, Sanders D, Krogan N, Shaw M, König R, Stertz S,

531 García-Sastre A, and Chanda Sumit K. 2015. Meta- and Orthogonal Integration of Influenza

532 “OMICs" Data Defines a Role for UBR4 in Virus Budding. Cell Host \& Microbe 18:723-735.

533 10.1016/j.chom.2015.11.002

534 Wang J, Quan X, Peng D, and Hu G. 2019. Long noncoding RNA DLEU1 promotes cell

535 proliferation of glioblastoma multiforme. Mol Med Rep 20:1873-1882. 10.3892/mmr.2019.10428

536 Wang P, Li X, and Xie Y. 2020a. B4GalT1 Regulates Apoptosis and Autophagy of Glioblastoma 537 In Vitro and In Vivo. Technol Cancer Res Treat 19:1533033820980104.

$538 \quad 10.1177 / 1533033820980104$

539 Wang W, Xing H, Huang C, Pan H, and Li D. 2020b. Identification of pancreatic cancer type

540 related factors by Weighted Gene Co-Expression Network Analysis. Med Oncol 37:33.

541 10.1007/s12032-020-1339-0

542 Wu J, Su HK, Yu ZH, Xi SY, Guo CC, Hu ZY, Qu Y, Cai HP, Zhao YY, Zhao HF, Chen FR, 543 Huang YF, To ST, Feng BH, Sai K, Chen ZP, and Wang J. 2020. Skp2 modulates proliferation, 544 senescence and tumorigenesis of glioma. Cancer Cell Int 20:71. 10.1186/s12935-020-1144-Z

545 Xing Z-y, Sun L-g, and Guo W-j. 2015. Elevated expression of Notch-1 and EGFR induced

546 apoptosis in glioblastoma multiforme patients. Clinical Neurology and Neurosurgery 131:54-58.

547 10.1016/j.clineuro.2015.01.018

548 Zhang B, and Horvath S. 2005. A general framework for weighted gene co-expression network

549 analysis. Stat Appl Genet Mol Biol 4:Article17. 10.2202/1544-6115.1128

550 Zhang L, Zhang Z, and Yu Z. 2019. Identification of a novel glycolysis-related gene signature

551 for predicting metastasis and survival in patients with lung adenocarcinoma. J Transl Med

552 17:423. 10.1186/s12967-019-02173-2

553 Zhao J, Liu B, Yang JA, Tang D, Wang X, and Chen Q. 2019. Human sperm-associated antigen

5544 as a potential biomarker of glioblastoma progression and prognosis. Neuroreport 30:446-451.

555 10.1097/WNR.0000000000001226

556 Zheng W, Zou Z, Lin S, Chen X, Wang F, Li X, and Dai J. 2018. Identification and functional

557 analysis of spermatogenesis-associated gene modules in azoospermia by weighted gene

558 coexpression network analysis. Journal of Cellular Biochemistry 120:3934-3944.

$559 \quad 10.1002 / j \mathrm{cb} .27677$

560 Zhou Z, Cheng Y, Jiang Y, Liu S, Zhang M, Liu J, and Zhao Q. 2018. Ten hub genes associated

561 with progression and prognosis of pancreatic carcinoma identified by co-expression analysis.

562 International Journal of Biological Sciences 14:124-136. 10.7150/ijbs.22619

563 Zucchini C, Strippoli P, Biolchi A, Solmi R, Lenzi L, D'Addabbo P, Carinci P, and Valvassori L.

564 2003. The human TruB family of pseudouridine synthase genes, including the Dyskeratosis

565 Congenita 1 gene and the novel member TRUB1. Int J Mol Med 11:697-704.

566 Zurlo G, and Zhang Q. 2020. Adenylosuccinate lyase hydroxylation contributes to triple negative

567 breast cancer via the activation of cMYC. Mol Cell Oncol 7:1707045.

$56810.1080 / 23723556.2019 .1707045$ 


\section{Figure Caption}

572 Fig. 1. Identification of gene co-expression modules. (A) cluster samples to detect outliers. All

573 samples are located in the cluster and pass the critical threshold at the same time. The blue

574 highlighting means that the samples are in strong trait relationships established by correlation

575 analyses. (B) The scale-free fit index was analyzed under the background of different soft-

576 thresholding power $(\beta)$.(C) Analyze average connectivity when using different soft-thresholding

577 powers.(D) Dendrogram clustering of all genomic genes in GBM samples.(E) Heatmap of co-

578 expressed genes.Different modules on the $\mathrm{X}$ and $\mathrm{Y}$ axis have different colors. The connection

579 degree of different modules is indicated by the yellow intensity.

580 Fig. 2. Correlation of modules and clinical traits. (A) Module intrinsic genes and relapse

581 Heatmap of the correlation between. (B) Scatter plot of the correlation between the darkturquoise 582 module and relapse. All modules can be correlated with genes, and all continuous traits can be

583 correlated with gene expression values. The two correlation matrices are combined and the

584 vertical coordinate is the Gene significance for relapse when the module of interest is specified

585 for analysis. "Module membership" as "correlation in expression between the given gene with

586 the eigengene of the module" .(C) Enrichment analysis of the function and pathway of the

587 darkturquoise module. The rich biological process terms in the selected modules are described as

588 interactive networks and listed according to their P-value. The size of the dots represents the

589 number of genes that are co-expressed, the larger the dot, the more genes are co-expressed,

590 presumably the more important they are and the more important they are as core genes in the

591 network graph. Each node is a gene. The size of the node means degree of gene enrichment. Set

$592 \mathrm{P}<0.01$ as the cutoff criterion.Enrich the term network, colored with cluster-ID, where nodes

593 sharing the same cluster ID are usually close to each other.

594 Fig. 3. The Kaplan-Meier survival curve can evaluate the prognostic performance of core 595 genes based on the expression status of selected biomarkers in the database. (A) DARS. (B)

596 GDI2. (C) P4HA2. (D)TRUB1. All patients in each group were divided into high expression

597 group and low expression group by gene expression. The cutoff for low versus high expression is 598 3-fold expression of controls.

599 Fig. 4. Gene co-expression. (A-H) In the TCGA dataset, selected the mRNA expression levels 600 of DARS / GDI2 / P4HA2 / TRUB1 related genes, analyzed the correlation of these genes

601 through $\mathrm{R}$, and visualize them with the circus and heatmap graph. (I-L) The four genes with the 602 highest correlation with DARS / GDI2 / P4HA2 / TRUB1, drew scatter plots.

603 Fig. 5. Drug sensitivity analysis. (A) The role of DARS/GDI2/P4HA2/TRUB1 in the famous 604 cancer related pathways (GSCALite). The size of an area in the pie chart represents the extent of 605 the role of DARS/GDI2/P4HA2/TRUB1 in the well-known cancer-related pathway (GSCALite).

606 (B) In the GDSC training set,high expression of DARS/GDI2/P4HA2/TRUB1 was inferred to be 607 less sensitive to commonly used chemotherapy drugs. The test for association between paired 
608 samples used Pearson's correlation coefficient. Two-tailed statistical P values were calculated by

609 a two-sample Mann-Whitney test or Student's t test when appropriate.

610 Fig. 6. Genetic and transcriptional changes and connections with immune cell populations.

611 (A) Correlation of DARS/GDI2/P4HA2/TRUB1 expression with immune infiltration level in 612 GBM. (B) DARS/GDI2/P4HA2/TRUB1 copy number alterations (CNV) affects the level of

613 infiltration of B cells, CD8+ T cells, CD4+T celles, Macrophages, Neutrophils, and Dendritic 614 cells in GBM.

615 Fig. 7. Genomic alterations of DARS/GDI2/P4HA2/TRUB1 in GBM. OncoPrint of 616 DARS/GDI2/P4HA2/TRUB1 alterations in GBM cohort. The different types of genetic 617 alterations are highlighted in different colors. Expression profiles of mRNAs showing different 618 expression ( $\geq 3$-fold) compared to control were considered to be mRNA high, and vice versa for 619 low.

620 Fig. 8. GSVA analysis. GSVA of DARS/GDI2/P4HA2/TRUB1 gene sets in GBM. (A) 621 DARS. (B) GDI2. (C) P4HA2. (D) TRUB1.A t value $>1$ or $<-1$ represents statistically 622 significant changes.

623 


\section{Figure 1}

Identification of gene co-expression modules.

(A) cluster samples to detect outliers. All samples are located in the cluster and pass the critical threshold at the same time. The green highlighting means that the samples are in strong trait relationships established by correlation analyses. (B) The scale-free fit index was analyzed under the background of different soft-thresholding power ( $\beta$ ).(C) Analyze average connectivity when using different soft-thresholding powers.(D) Dendrogram clustering of all genomic genes in GBM samples.(E) Heatmap of co-expressed genes. Different modules on the $X$ and $Y$ axis have different colors. The connection degree of different modules is indicated by the yellow intensity. 

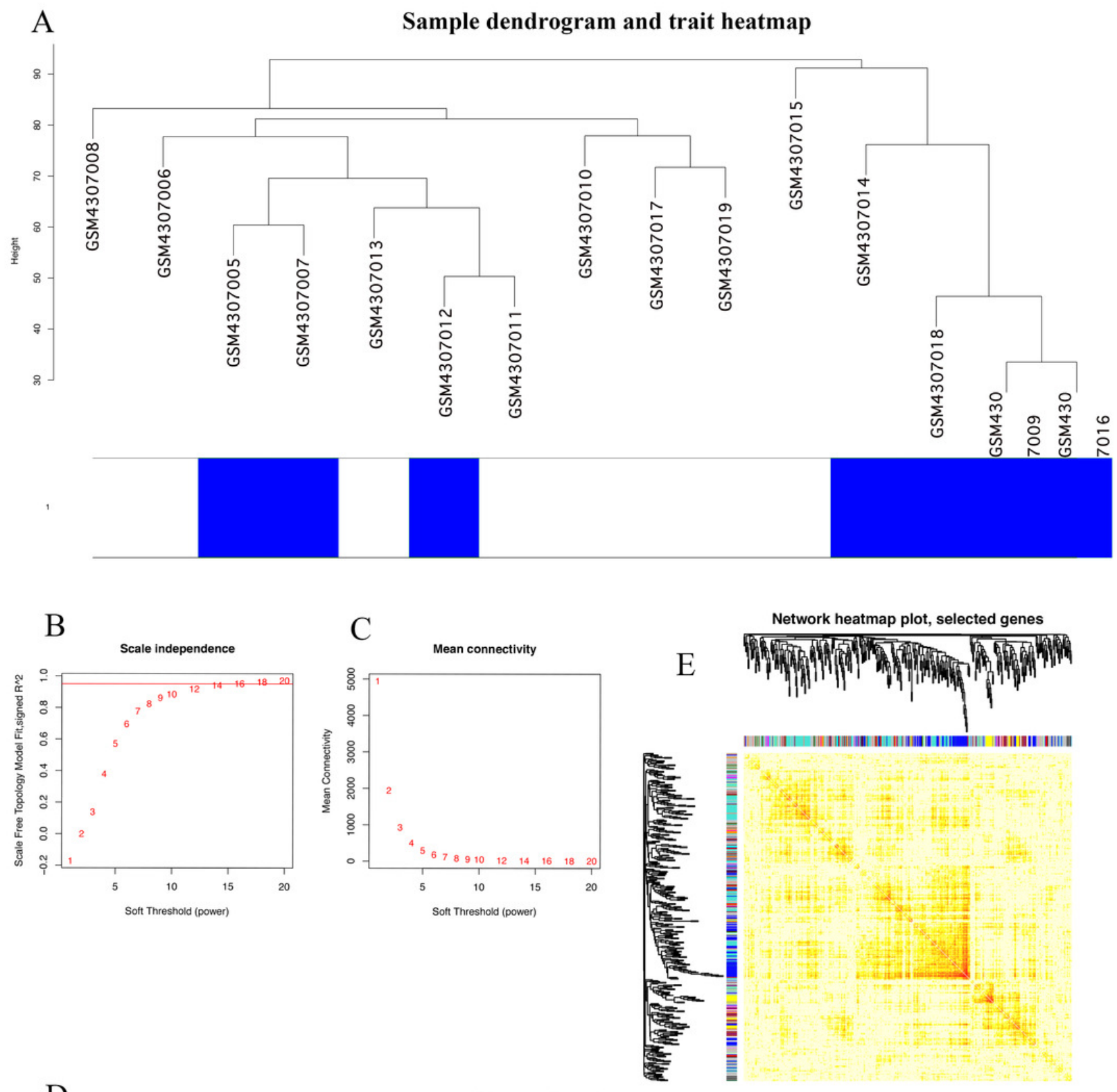

D

Cluster Dendrogram

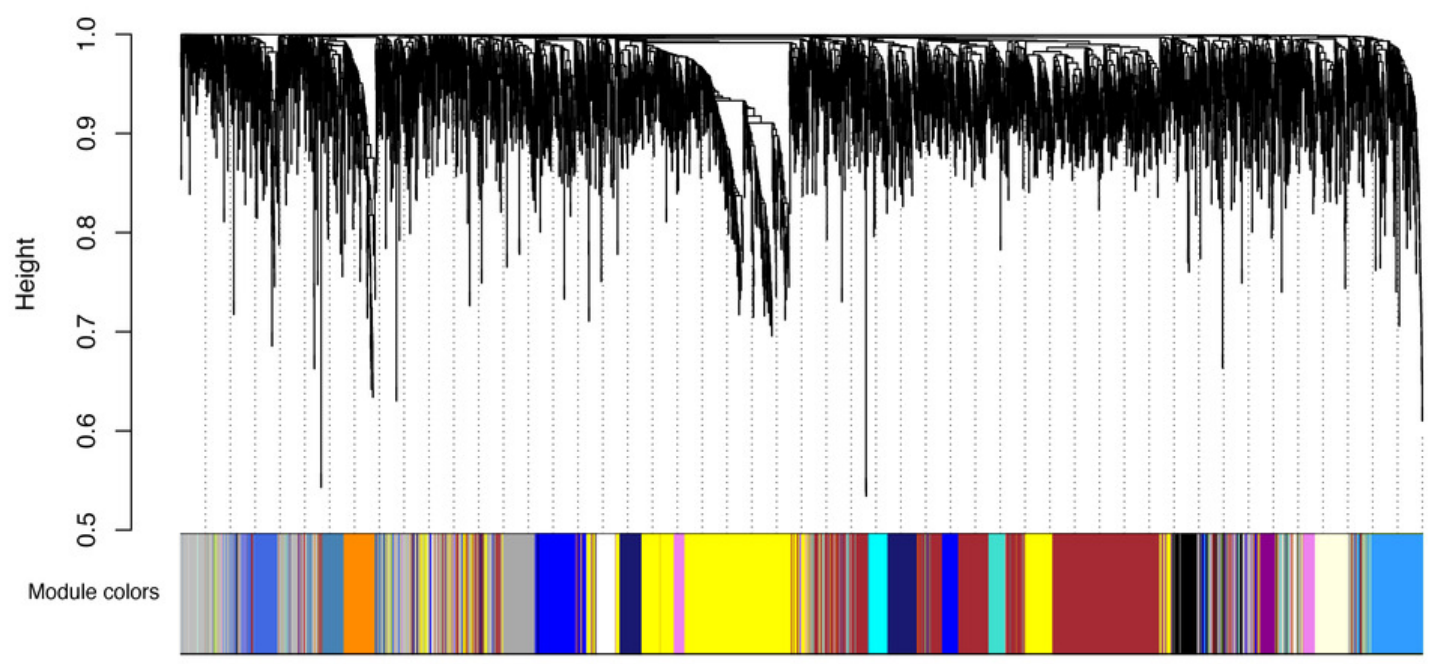




\section{Figure 2}

Correlation of modules and clinical traits.

(A) Module intrinsic genes and relapse Heatmap of the correlation between. (B) Scatter plot of the correlation between the darkturquoise module and relapse. All modules can be correlated with genes, and all continuous traits can be correlated with gene expression values. The two correlation matrices are combined and the vertical coordinate is the Gene significance for luminal when the module of interest is specified for analysis. (C) Enrichment analysis of the function and pathway of the darkturquoise module.The rich biological process terms in the selected modules are described as interactive networks and listed according to their P-value. The size of the dots represents the number of genes that are co-expressed, the larger the dot, the more genes are co-expressed, presumably the more important they are and the more important they are as core genes in the network graph. Each node is a gene. The size of the node means degree of gene enrichment. Set $P<0.01$ as the cutoff criterion.Enrich the term network, colored with cluster-ID, where nodes sharing the same cluster ID are usually close to each other. 

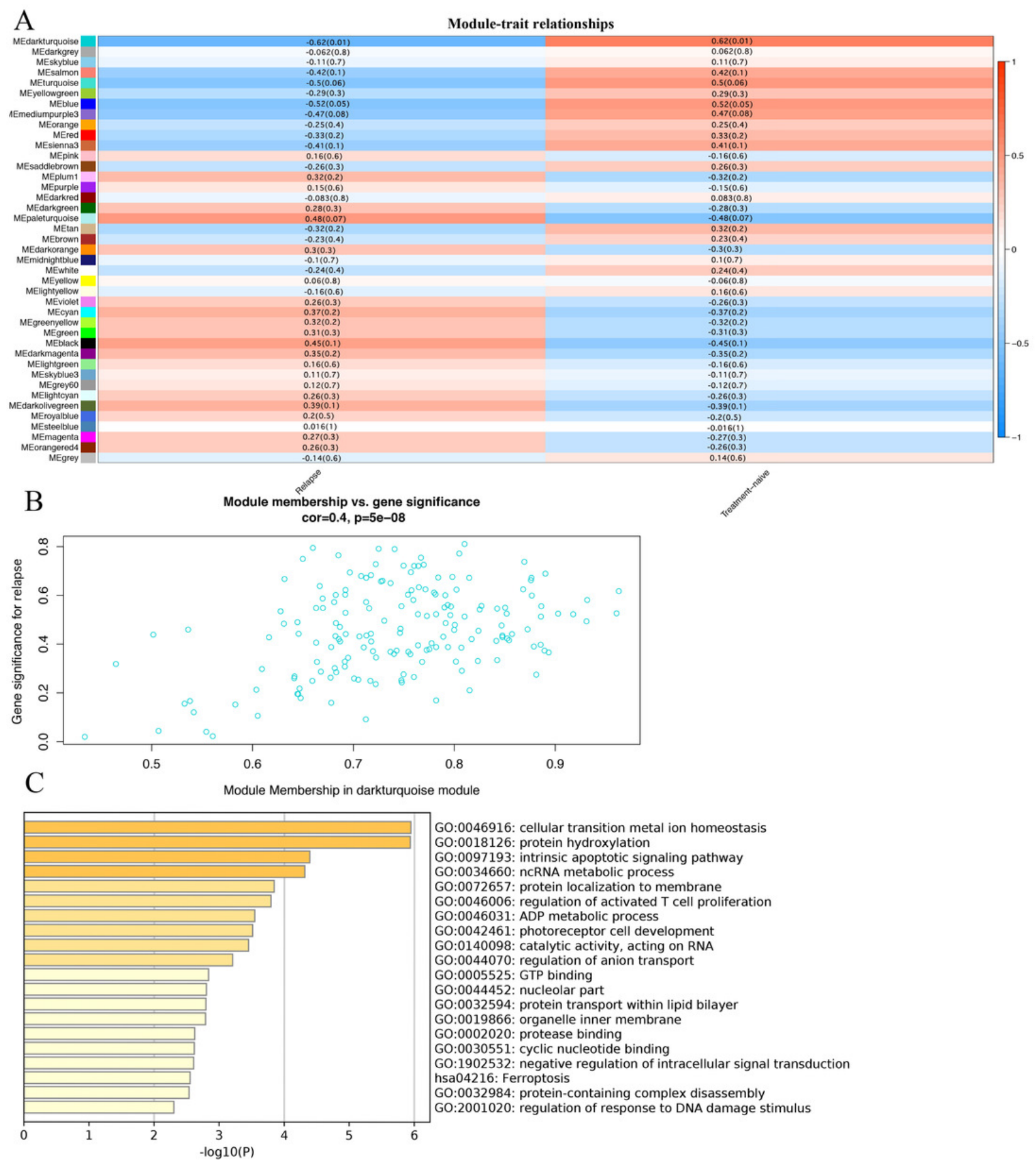

GO:0046916: cellular transition metal ion homeostasis GO:0018126: protein hydroxylation

GO:0097193: intrinsic apoptotic signaling pathway

GO:0034660: ncRNA metabolic process

GO:0072657: protein localization to membrane

GO:0046006: regulation of activated T cell proliferation

GO:0046031: ADP metabolic process

GO:0042461: photoreceptor cell development

GO:0140098: catalytic activity, acting on RNA

GO:0044070: regulation of anion transport

GO:0005525: GTP binding

GO:0044452: nucleolar part

GO:0032594: protein transport within lipid bilayer

GO:0019866: organelle inner membrane

GO:0019866: organelle inner m

GO:0030551: cyclic nucleotide binding

GO:1902532: negative regulation of intracellular signal transduction

hsa04216: Ferroptosis

GO:0032984: protein-containing complex disassembly

GO:2001020: regulation of response to DNA damage stimulus
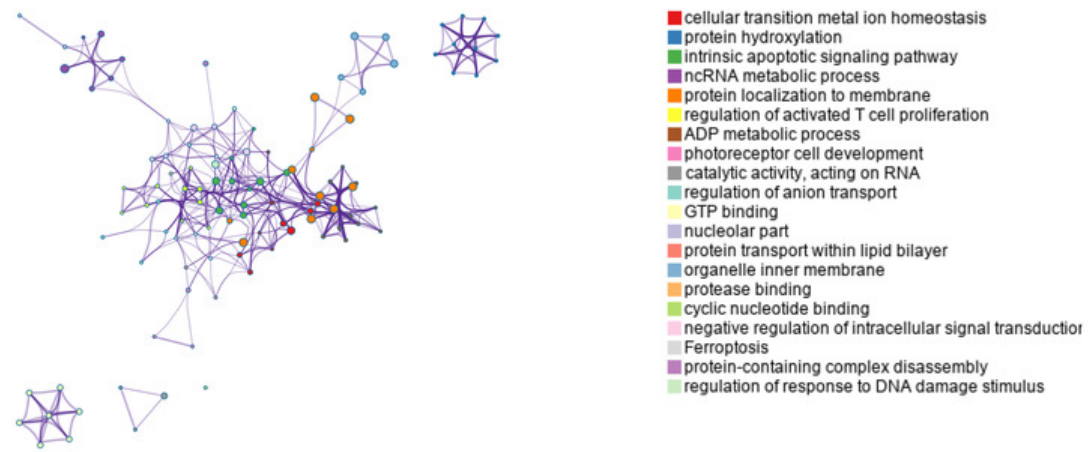
Figure 3

The Kaplan-Meier survival curve can evaluate the prognostic performance of core genes based on the expression status of selected biomarkers in the database.

(A) DARS. (B) GDI2. (C) P4HA2. (D)TRUB1. All patients in each group were divided into high expression group and low expression group by gene expression. The cutoff for low versus high expression is 3-fold expression of controls. 
A

Survival curve $(p=4.271 e-02)$

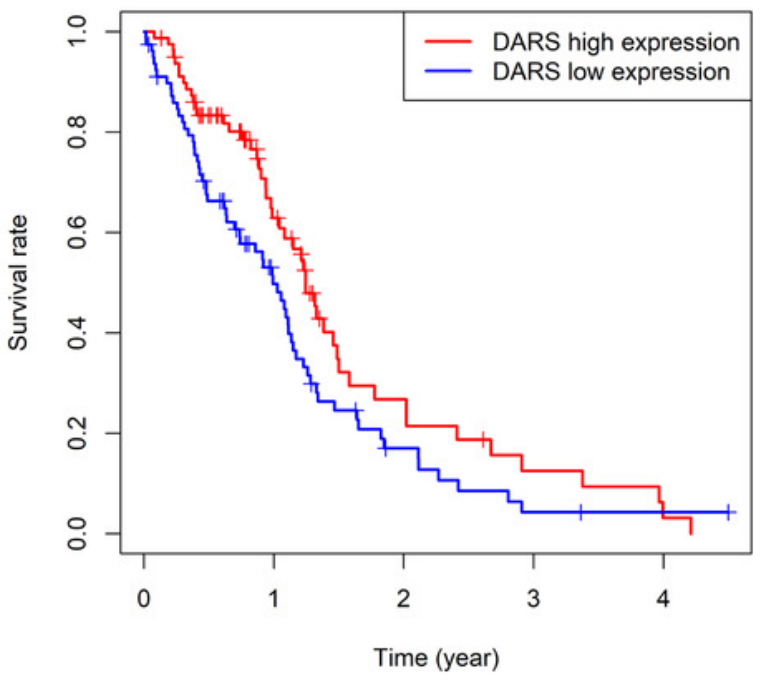

C

Survival curve $(p=1.019 e-02)$

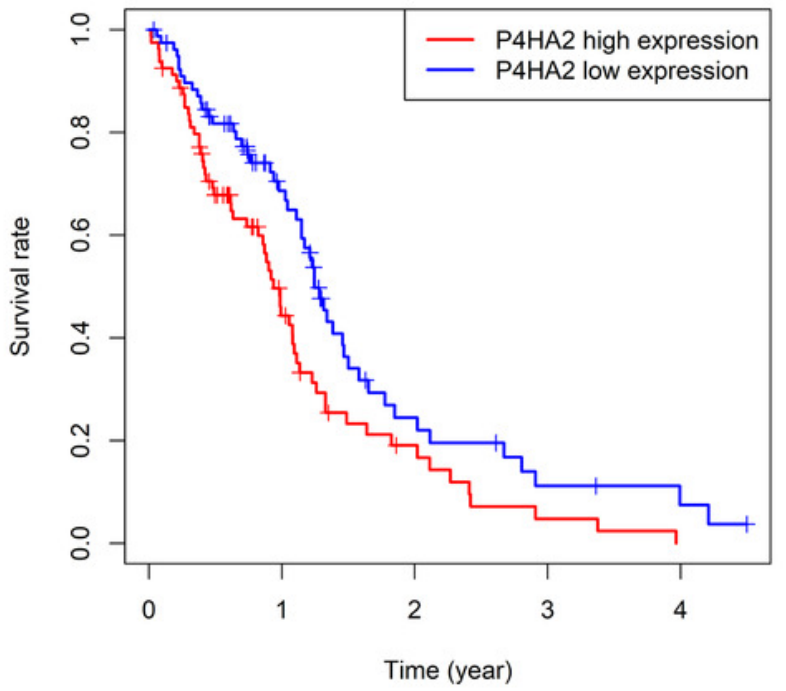

B

Survival curve $(p=4.42 e-03)$

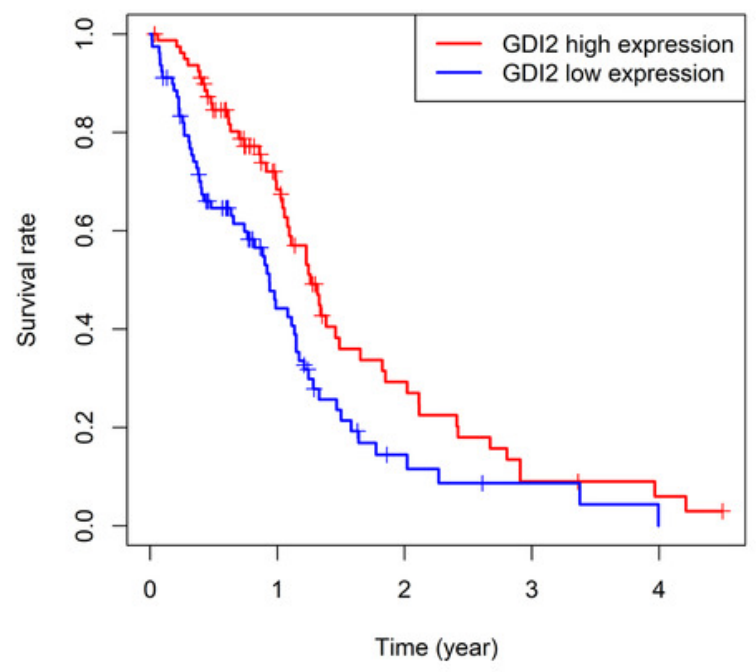

D

Survival curve $(p=2.247 e-02)$

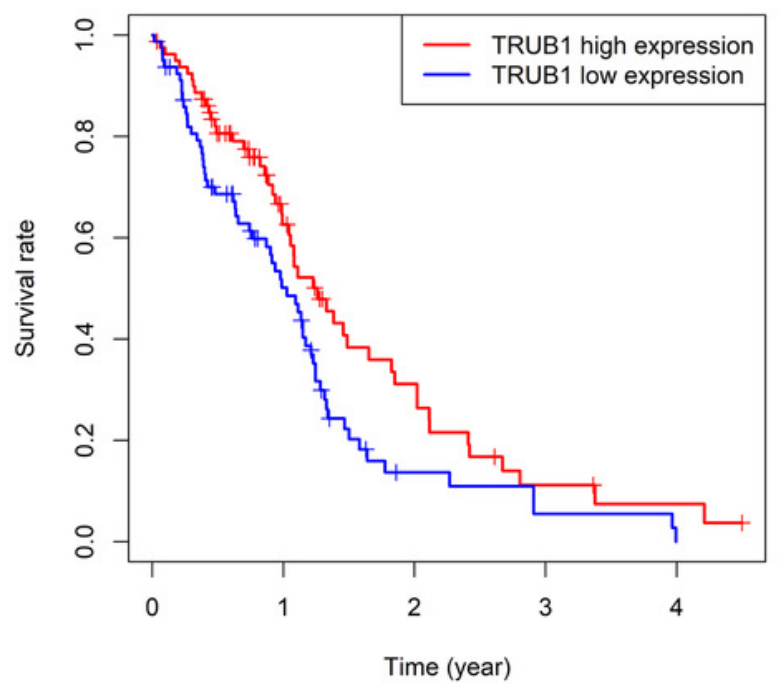


Figure 4

Gene co-expression.

(A-H) In the TCGA dataset, selected the mRNA expression levels of DARS / GDI2 / P4HA2 /

TRUB1 related genes, analyzed the correlation of these genes through $R$, and visualize them with the circus and heatmap graph. (I-L) The four genes with the highest correlation with DARS / GDI2 / P4HA2 / TRUB1, drew scatter plots.
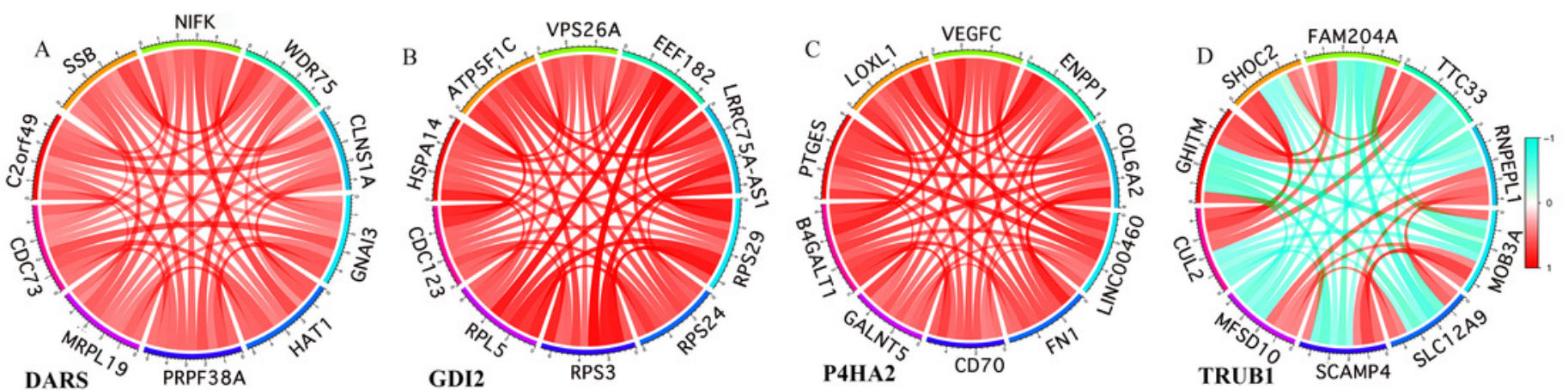

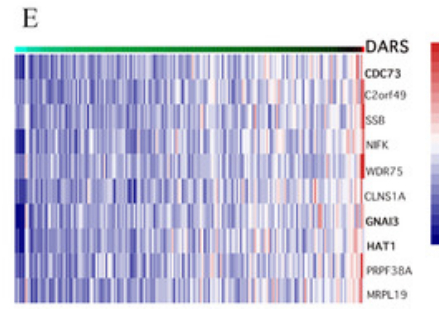

DARS
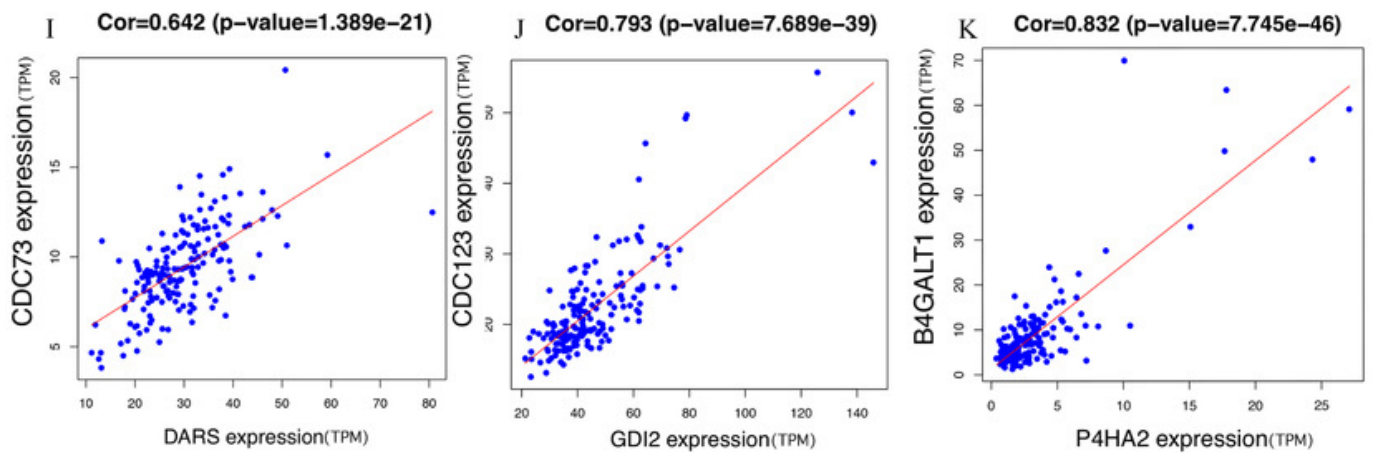
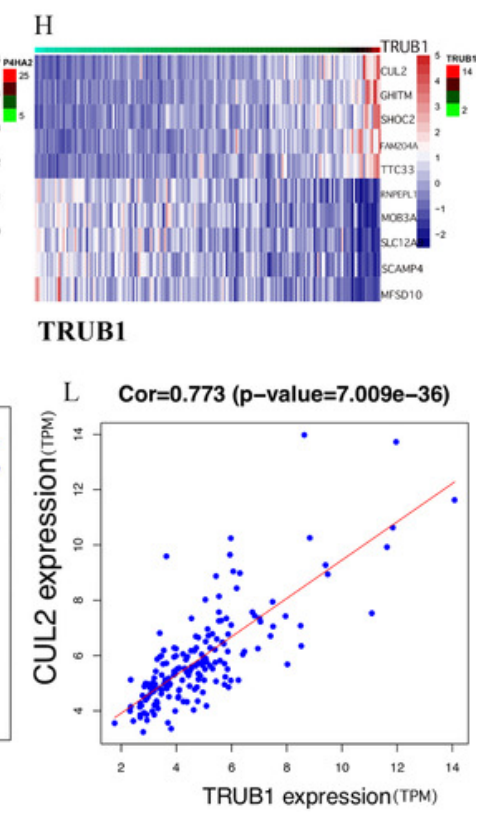


\section{Figure 5}

Drug sensitivity analysis.

(A) The role of DARS/GDI2/P4HA2/TRUB1 in the famous cancer related pathways (GSCALite).

The size of an area in the pie chart represents the extent of the role of

DARS/GDI2/P4HA2/TRUB1 in the well-known cancer-related pathway (GSCALite). (B) In the GDSC training set,high expression of DARS/GDI2/P4HA2/TRUB1 was inferred to be less sensitive to commonly used chemotherapy drugs. The test for association between paired samples used Pearson's correlation coefficient. Two-tailed statistical P values were calculated by a two-sample Mann-Whitney test or Student's t test when appropriate. 


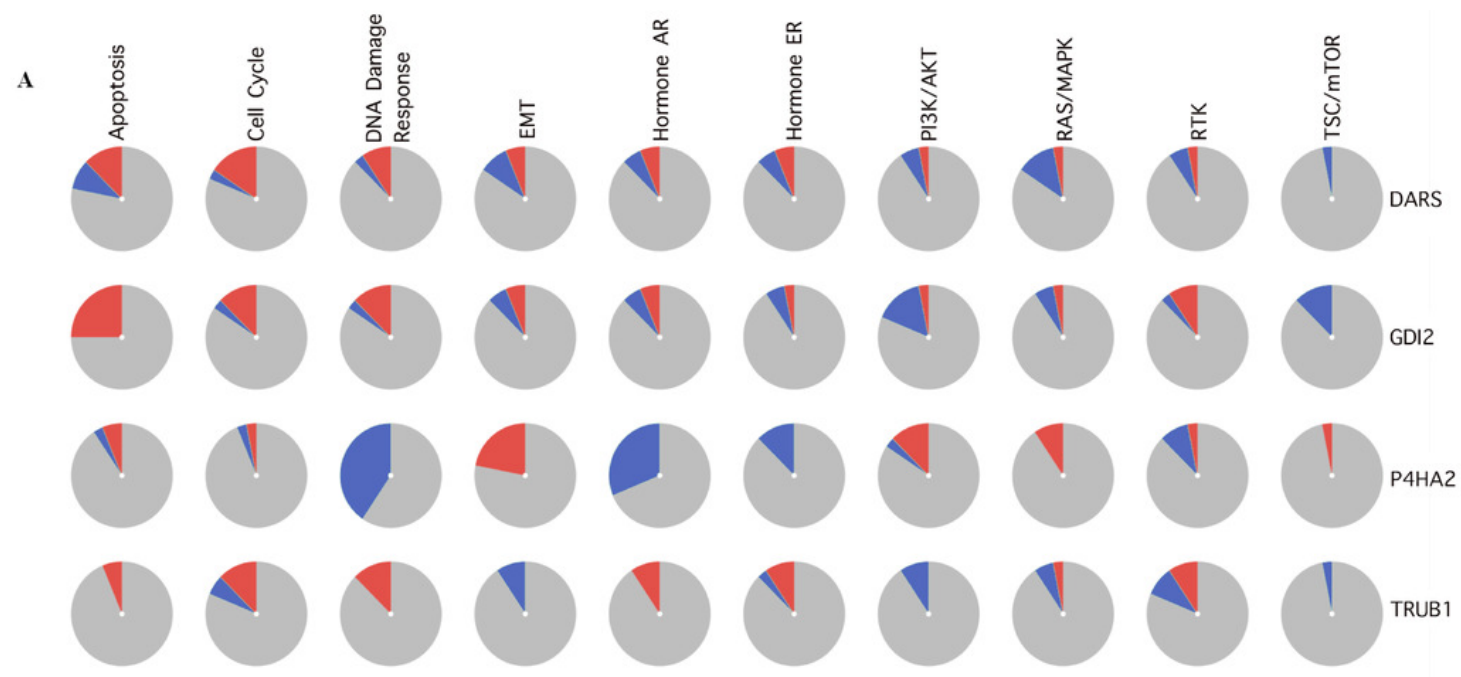

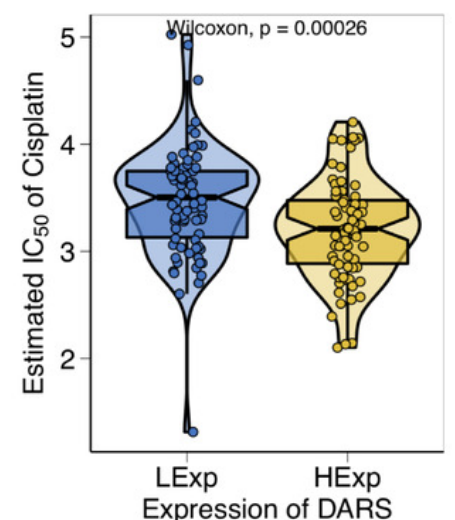
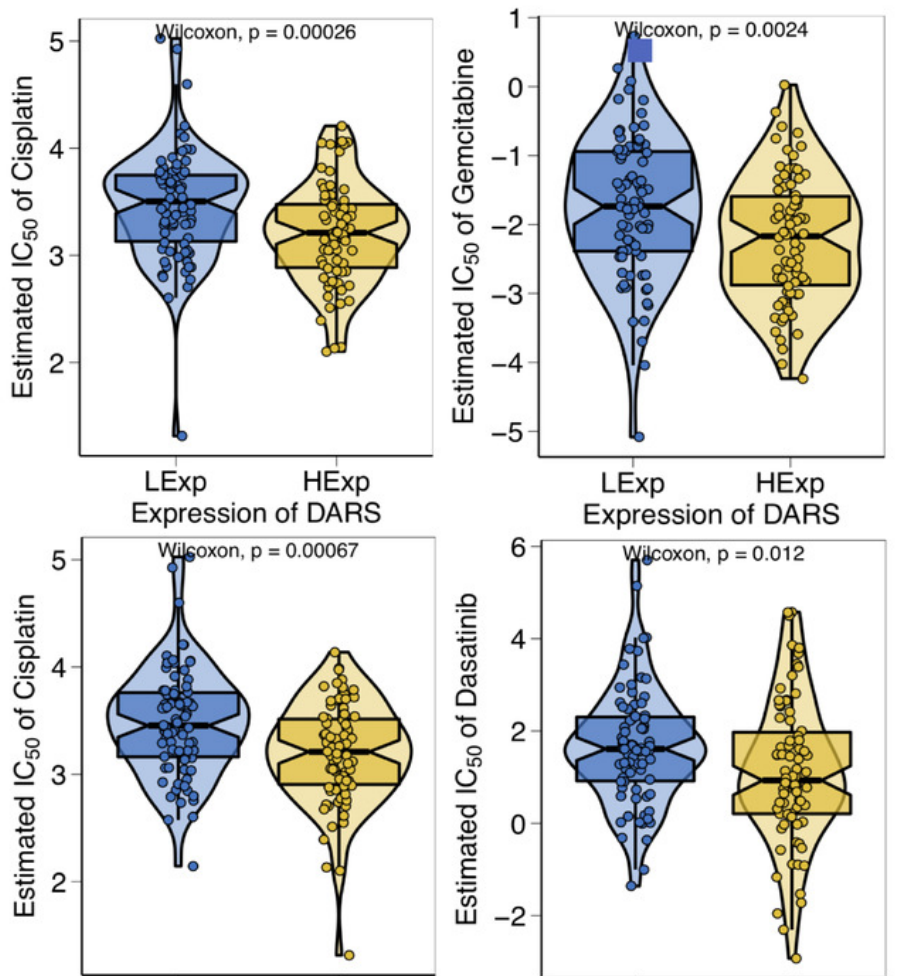

LExp HExp

Expression of GDI2
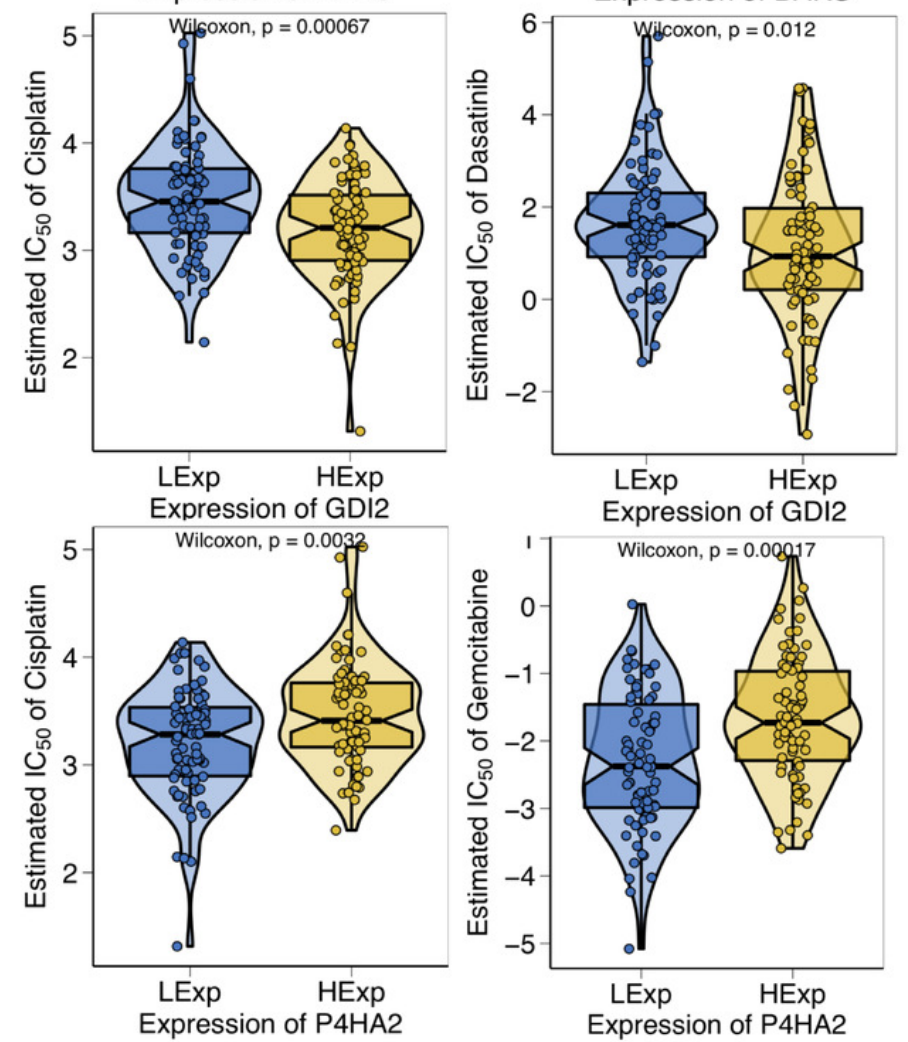

Expression of GDI2

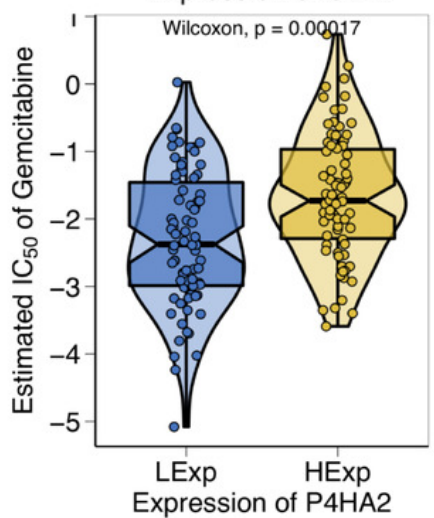

$\square$ Activation $\square$ Inhibition $\square$ None
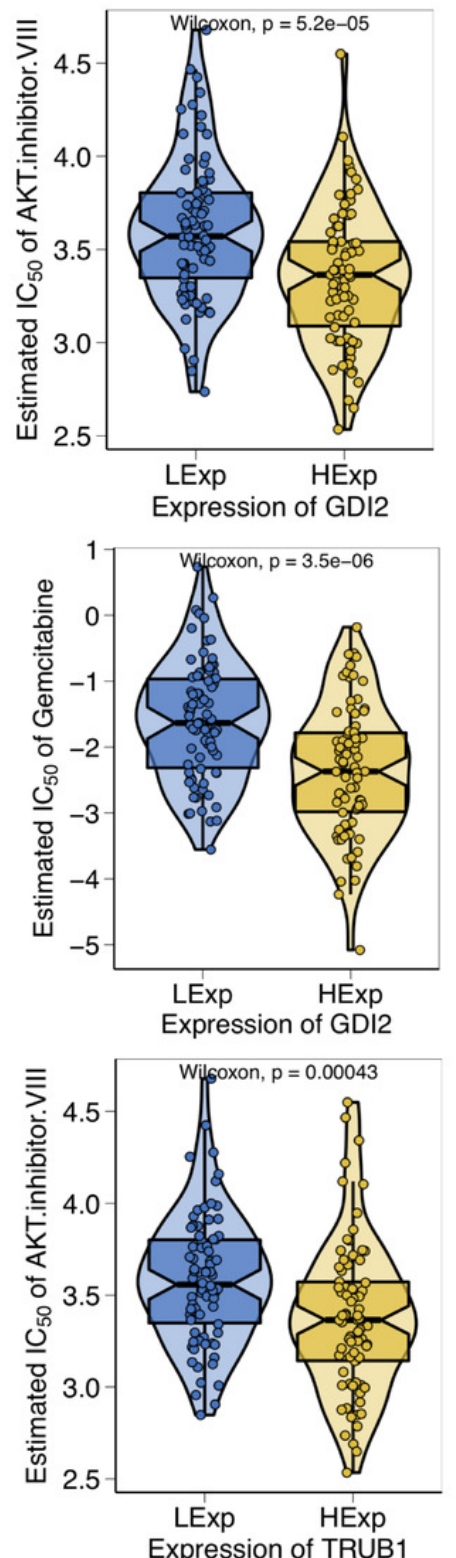


\section{Figure 6}

Genetic and transcriptional changes and connections with immune cell populations.

(A) Correlation of DARS/GDI2/P4HA2/TRUB1 expression with immune infiltration level in GBM.

(B) DARS/GDI2/P4HA2/TRUB1 copy number alterations (CNV) affects the level of infiltration of B cells, CD8+ T cells, CD4+T celles, Macrophages, Neutrophils, and Dendritic cells in GBM. 

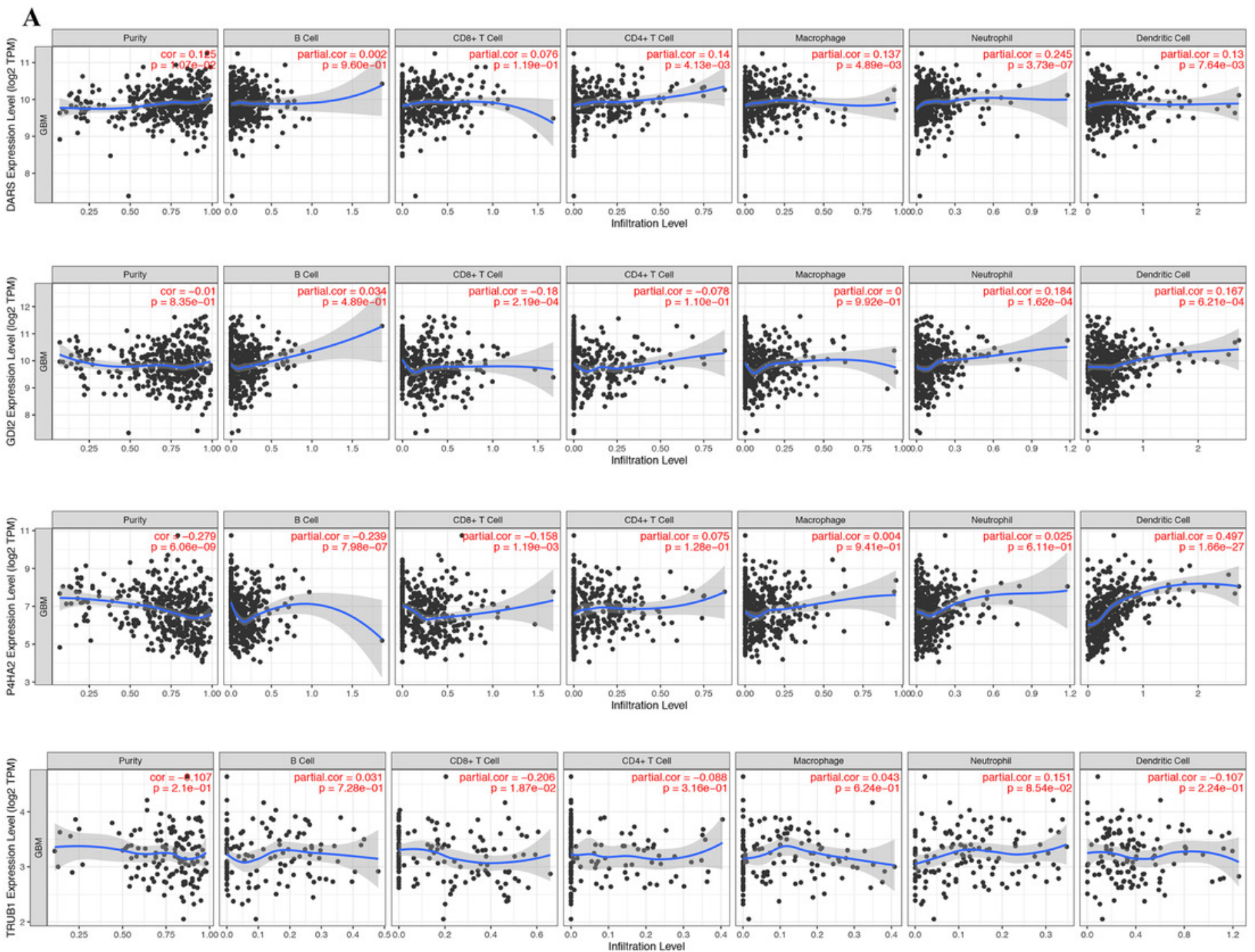

B

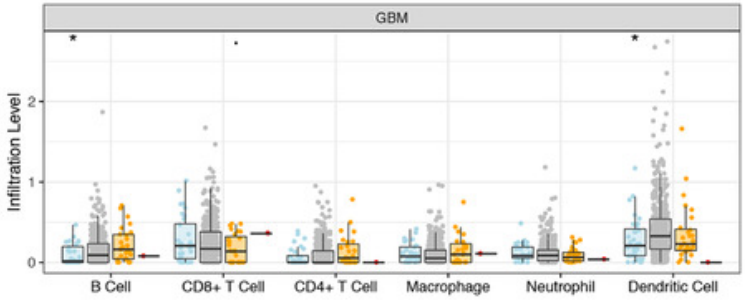

DARS

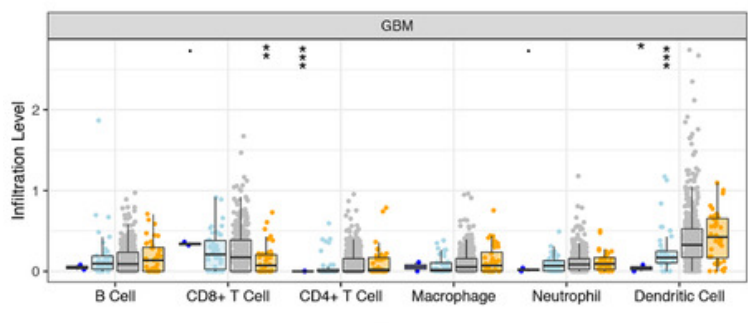

P4HA2

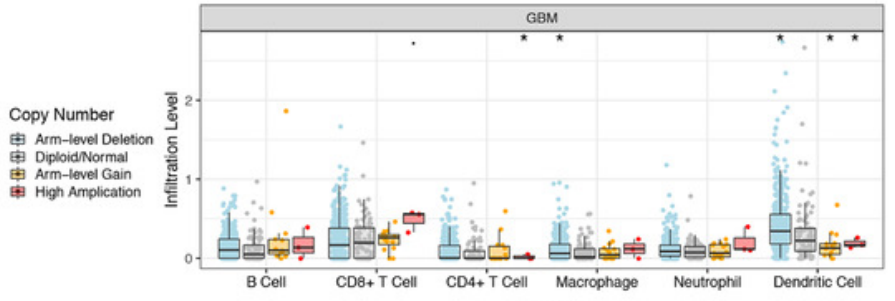

GDI2

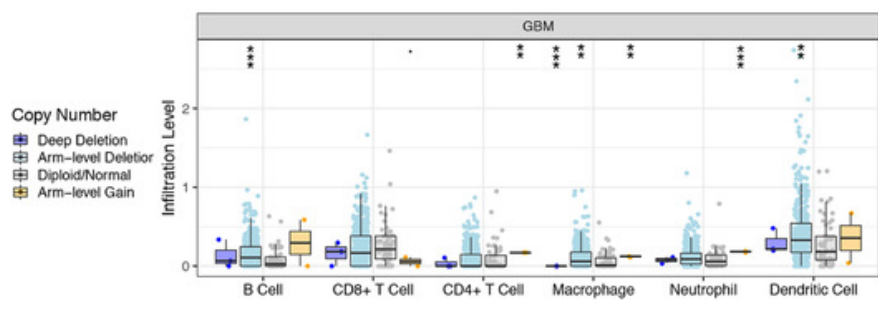

Copy Number

由er Deep Deletion
in Arm-level Deletion

由 Diploidnormal 


\section{Figure 7}

Genomic alterations of DARS/GDI2/P4HA2/TRUB1 in GBM.

OncoPrint of DARS/GDI2/P4HA2/TRUB1 alterations in GBM cohort. The different types of genetic alterations are highlighted in different colors. Expression profiles of mRNAs showing different expression ( $\geq 3$-fold) compared to control were considered to be mRNA high, and vice versa for low.

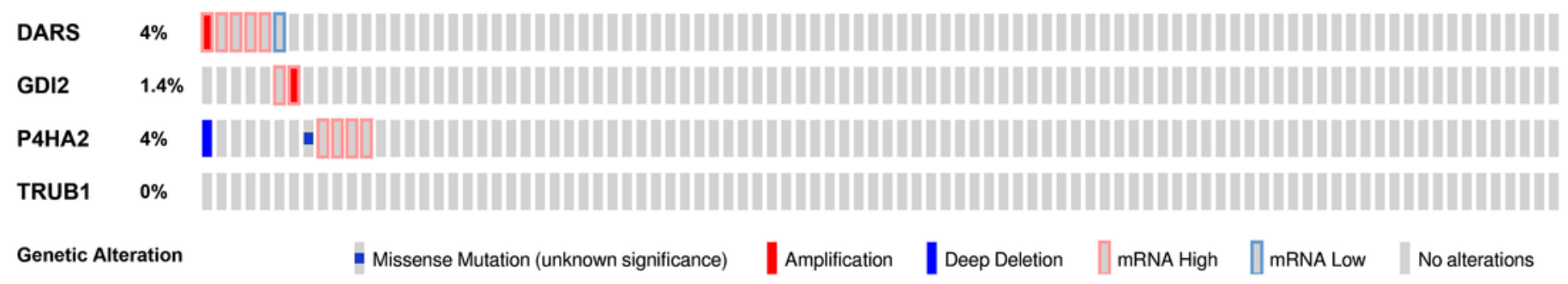


Figure 8

Fig. 8. GSVA analysis. GSVA of DARS/GDI2/P4HA2/TRUB1 gene sets in GBM.

(A) DARS. (B) GDI2. (C) P4HA2. (D) TRUB1.A t value $>1$ or $<-1$ represents statistically significant changes. 
DARS

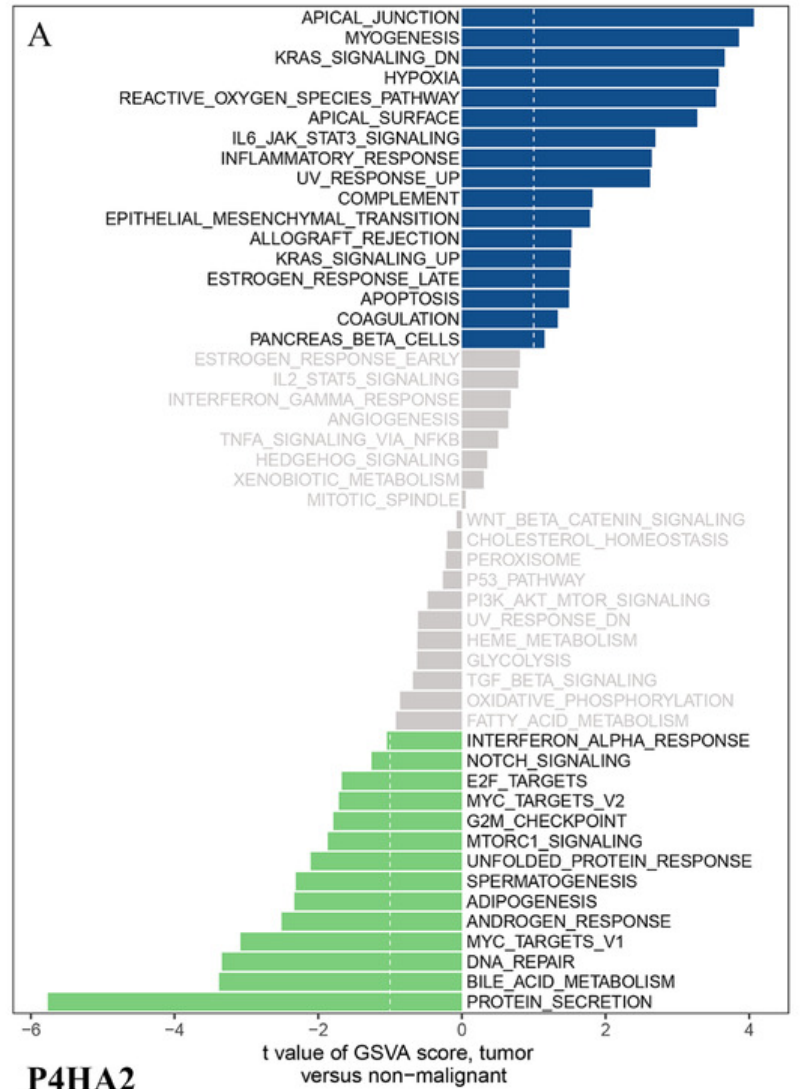

\section{P4HA2}

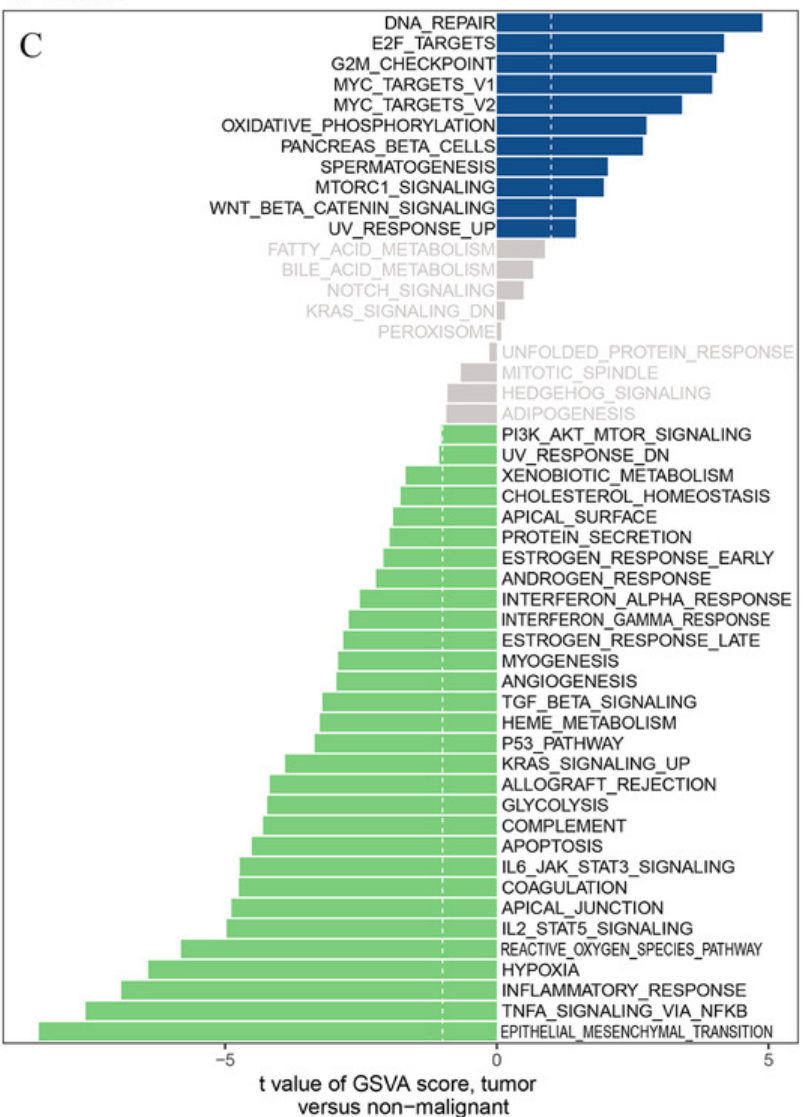

GDI2
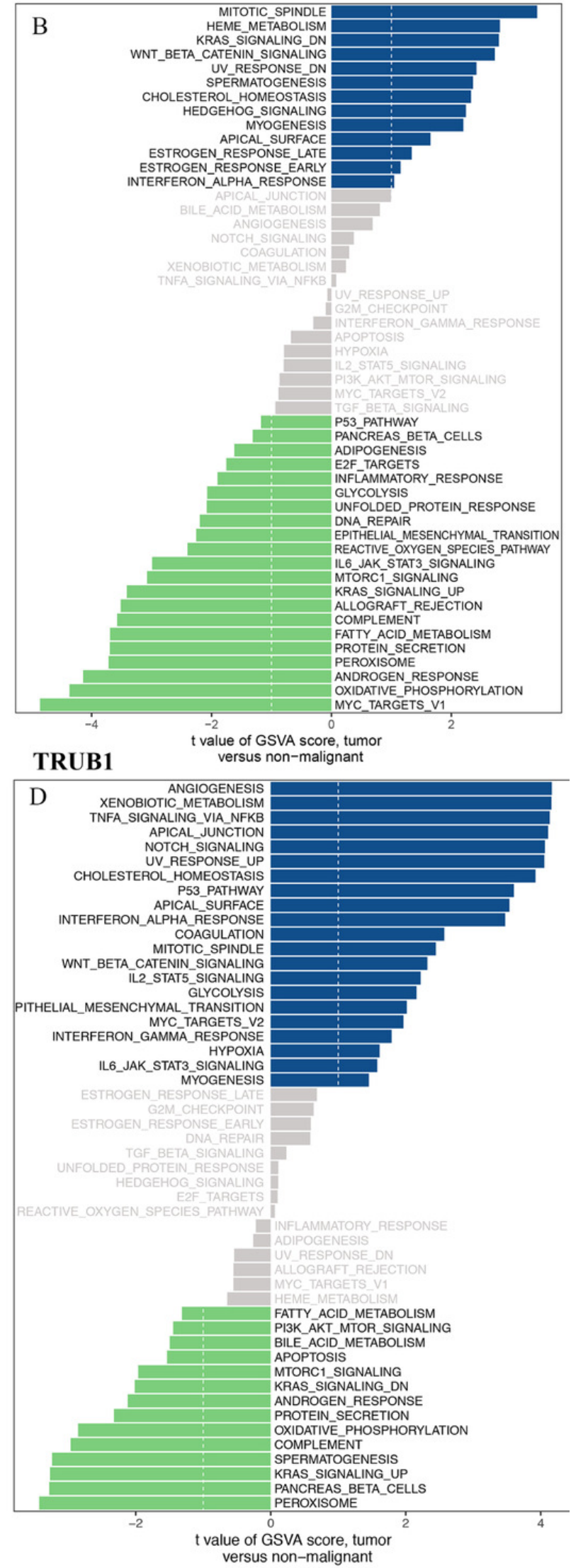


\section{Table $\mathbf{1}$ (on next page)}

Table1: Statistics of genes in darkturquoise modules.

Table1 Statistics of genes in darkturquoise modules. 
1 Table1 Statistics of genes in darkturquoise modules.

2

\begin{tabular}{ll}
\hline Gene & P value \\
\hline TRUB1 & $2.25 \mathrm{E}-02$ \\
P4HA2 & $1.02 \mathrm{E}-02$ \\
DARS & $4.27 \mathrm{E}-02$ \\
FKBP1B & $6.13 \mathrm{E}-03$ \\
NRL & $2.20 \mathrm{E}-02$ \\
CORO6 & $1.83 \mathrm{E}-02$ \\
LRRC43 & $4.65 \mathrm{E}-02$ \\
GAS6 & $3.63 \mathrm{E}-02$ \\
SPAG4 & $2.07 \mathrm{E}-03$ \\
PRKAR2B & $1.48 \mathrm{E}-02$ \\
CAMSAP2 & $1.31 \mathrm{E}-02$ \\
CD24 & $2.52 \mathrm{E}-02$ \\
GDI2 & $4.42 \mathrm{E}-03$ \\
DLEU1 & $1.45 \mathrm{E}-02$ \\
\hline
\end{tabular}

3 